This item was submitted to Loughborough's Research Repository by the author.

Items in Figshare are protected by copyright, with all rights reserved, unless otherwise indicated.

\title{
A plethora of three-dimensional periodic travelling gravity-capillary water waves with multipulse transverse profiles
}

PLEASE CITE THE PUBLISHED VERSION

LICENCE

CC BY-NC-ND 4.0

\section{REPOSITORY RECORD}

Groves, Mark D., and B. Sandstede. 2019. "A Plethora of Three-dimensional Periodic Travelling Gravitycapillary Water Waves with Multipulse Transverse Profiles”. figshare. https://hdl.handle.net/2134/304. 


\title{
A plethora of three-dimensional periodic travelling gravity-capillary water waves with multipulse transverse profiles
}

\author{
M. D. Groves \\ Department of Mathematical Sciences \\ Loughborough University \\ Loughborough \\ LE11 3TU \\ United Kingdom
}

\author{
B. Sandstede \\ Department of Mathematics \\ Ohio State University \\ Columbus \\ Ohio 43210 \\ USA
}

\begin{abstract}
This article presents a rigorous existence theory for three-dimensional gravity-capillary water waves which are uniformly translating and periodic in one spatial direction $x$ and have the profile of a uni- or multipulse solitary wave in the other $z$. The waves are detected using a combination of Hamiltonian spatial dynamics and homoclinic Lyapunov-Schmidt theory.

The hydrodynamic problem is formulated as an infinite-dimensional Hamiltonian system in which $z$ is the time-like variable, and a family of points $P_{k, k+1}, k=1,2, \ldots$ in its two-dimensional parameter space is identified at which a Hamiltonian $0^{2} 0^{2}$ resonance takes place (the zero eigenspace and generalised eigenspace are respectively two and four dimensional). The point $P_{k, k+1}$ is precisely that at which a pair of two-dimensional periodic linear travelling waves with frequency ratio $k: k+1$ simultaneously exist ('Wilton ripples'). A reduction principle is applied to demonstrate that the problem is locally equivalent to a four-dimensional Hamiltonian system near $P_{k, k+1}$.

It is shown that a Hamiltonian real semisimple $1: 1$ resonance, where two geometrically double real eigenvalues exist, arises along a critical curve $R_{k, k+1}$ emanating from $P_{k, k+1}$. Unipulse transverse homoclinic solutions to the reduced Hamiltonian system at points of $R_{k, k+1}$ near $P_{k, k+1}$ are found by a scaling and perturbation argument, and the homoclinic Lyapunov-Schmidt method is applied to construct an infinite family of multipulse homoclinic solutions which resemble multiple copies of the unipulse solutions.
\end{abstract}




\section{Introduction}

\subsection{Hamiltonian spatial dynamics and travelling water waves}

The classical three-dimensional gravity-capillary water wave problem concerns the irrotational flow of a perfect fluid of unit density subject to the forces of gravity and surface tension. The fluid motion is described by the Euler equations in a domain bounded below by a rigid horizontal bottom $\{Y=0\}$ and above by a free surface which is described as a graph $\{Y=h+\rho(x, z, t)\}$, where $h$ denotes the depth of the water in its undisturbed state and the function $\rho$ depends upon the two horizontal spatial directions $x, z$ and time $t$. Travelling waves are water waves which are uniformly translating in a distinguished horizontal direction without change of shape; without loss of generality we assume that the waves propagate in the $x$-direction with speed $c$ and continue to write $x$ as an abbreviation for $x-c t$. In terms of an Eulerian velocity potential $\phi(x, Y, z, t)$ the mathematical problem for travelling waves is to solve the equations

$$
\begin{array}{ll}
\phi_{x x}+\phi_{Y Y}+\phi_{z z}=0 & 0<Y<1+\rho, \\
\phi_{Y}=0 & \text { on } Y=0, \\
\phi_{Y}=\rho_{x} \phi_{x}+\rho_{z} \phi_{z}-\rho_{x} & \text { on } Y=1+\rho
\end{array}
$$

and

$$
\begin{aligned}
-\phi_{x}+ & \frac{1}{2}\left(\phi_{x}^{2}+\phi_{Y}^{2}+\phi_{z}^{2}\right)+\alpha \rho \\
& -\beta\left[\frac{\rho_{x}}{\sqrt{1+\rho_{x}^{2}+\rho_{z}^{2}}}\right]_{x}-\beta\left[\frac{\rho_{z}}{\sqrt{1+\rho_{x}^{2}+\rho_{z}^{2}}}\right]_{z}=0 \quad \text { on } Y=1+\rho
\end{aligned}
$$

(see Stoker [23, Sections 1 and 2.1]), in which we have introduced dimensionless variables. The equations involve two physical parameters $\alpha:=g h / c^{2}$ and $\beta:=\sigma / h c^{2}$, where $g$ and $\sigma$ are respectively the acceleration due to gravity and the coefficient of surface tension.

The phrase 'spatial dynamics' refers to an approach where a physical problem is formulated as a (typically ill-posed) evolutionary equation in which an unbounded spatial coordinate plays the role of the time-like variable. The method was introduced by Kirchgässner [16] and has become the basis for a wide range of existence theories for travelling water waves and other problems in the applied sciences. In a series of papers Kirchgässner [17] and Iooss \& Kirchgässner $[12,13]$ showed how the method may be applied to the two-dimensional travelling gravity-capillary water-wave problem in which the transverse spatial direction $z$ is absent; the direction $x$ of wave propagation is the time-like variable. Their method relies upon the pivotal observation that the evolutionary system in question is amenable to treatment using the reduction theorem of Mielke [19], which states that the (infinite-dimensional) evolutionary system has a finite-dimensional invariant manifold called the centre manifold which contains all its small, bounded solutions; the original problem is thus locally equivalent to a system of ordinary differential equations whose solution set can, in theory, be analysed. Iooss \& Kirchgässner used this technique to construct existence theories for several types of solitary waves (pulse-like waves which decay to the undisturbed state of the water far up- and downstream) and generalised solitary waves (pulse-like waves which decay to a small-amplitude periodic disturbances far up- and downstream). 
Many conservative problems admit a formulation as an infinite-dimensional Hamiltonian evolutionary system in which an unbounded spatial coordinate plays the role of time, and Mielke's reduction theorem, when applicable, yields a reduced Hamiltonian system of ordinary differential equations (cf. Mielke [20]). This 'Hamiltonian spatial dynamics' method was applied to the two-dimensional travelling gravity-capillary water-wave problem by Buffoni, Groves \& Toland [2] and Buffoni \& Groves [1] to construct multipulse solitary waves by means of existence theories for multipulse homoclinic solutions of the reduced Hamiltonian system. The reduced system is in both papers four-dimensional and its Hamiltonian structure is a crucial ingredient in the existence theories presented there.

Hamiltonian spatial dynamics and centre-manifold reduction techniques were extended to three-dimensional travelling gravity-capillary water waves by Groves \& Mielke [9] and Groves [6]. The former reference considers waves which are periodic in the transverse spatial direction $z$ and uses the longitudinal variable $x$ as the time-like variable, while the latter considers waves which are periodic in the $x$-direction and uses $z$ as the time-like variable. Both of these choices, which were motivated by similar studies of model equations by respectively Haragus-Courcelle \& Ilichev [10] and Haragus-Courcelle \& Pego [11], represent natural steps from two to three dimensions: the former includes all two-dimensional travelling waves as special cases, while the latter facilitates a discussion of the 'dimension-breaking' phenomenon in which two dimensional waves spontaneously lose their spatial inhomogeneity in the $z$-direction (see Groves, Haragus \& Sun [8]).

Of course we have the freedom to take any horizontal spatial coordinate as the time-like variable, and this observation has recently been explored in detail by Groves \& Haragus [7]. They took the horizontal spatial direction $X$ making an angle $\theta_{1}$ with the positive $x$-axis as the timelike variable and considered waves which are periodic in the direction $Z$ making an angle $\theta_{2}$ with the positive $x$-axis. By varying the angles $\theta_{1}, \theta_{2}$, together with the physical parameters $\beta, \alpha$ and the frequency $\nu$ of the waves in the $Z$-direction, Groves \& Haragus systematically compiled a complete catalogue of bifurcation scenarios which are found in the dynamics of the reduced Hamiltonian system; the catalogue is extensive, containing virtually any bifurcation or resonance known in Hamiltonian-systems theory. They also applied appropriate nonlinear existence theories to a representative sample of bifurcation scenarios, obtaining in particular periodic solutions of the reduced Hamiltonian system together with homoclinic connections both to the zero equilibrium and to periodic solutions. Periodic solutions to the reduced system correspond to doubly periodic water waves, and it is possible to obtain existence results for doubly periodic water waves with arbitrary fundamental domain in this fashion (cf. Craig \& Nicholls [4], who obtain the same result by a different method). Homoclinic solutions to the reduced system correspond to water waves which have a solitary- or generalised solitary-wave profile in the $X$-direction and are periodic in the $Z$-direction.

\subsection{The mathematical framework}

In this paper we use the framework developed by Groves [6], where the hydrodynamic problem (1)-(4) is formulated as an infinite-dimensional Hamiltonian system in which the unbounded spatial coordinate $z$ plays the role of time and the variables $\rho, \phi$ are periodic in the $x$-direction with fixed frequency $\nu$. We proceed by fixing 'reference values' $\beta_{0}, \alpha_{0}$ of the physical parameters

$\beta$ and $\alpha$ and perturbing around them with a bifurcation parameter $\epsilon \in \mathbb{R}^{2}$; Mielke's reduction 


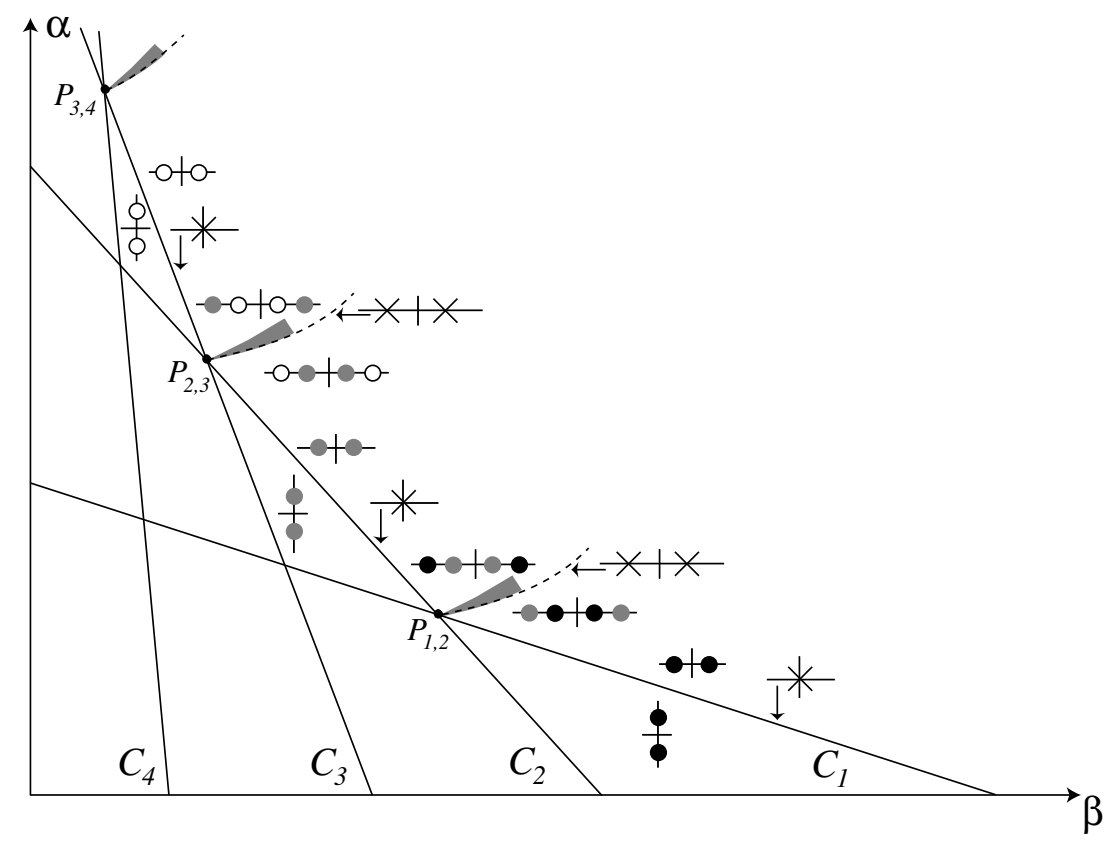

Figure 1: The line $C_{k}$ consists of points in $(\beta, \alpha)$-parameter space at which two real mode $k$ eigenvalues become purely imaginary by passing through zero in a non-semisimple resonance, and real mode $k$ and $k+1$ eigenvalues pass through each other in a semisimple resonance at the points on the dashed lines. Mode 1, 2 and 3 eigenvalues are represented respectively by black, grey and white dots. The shaded regions indicate the parameter regimes where multipulse homoclinic bifurcation is detected.

result yields a centre manifold whose dimension is equal to the number of purely imaginary eigenvalues at criticality, and the reduced Hamiltonian system valid for values of the bifurcation parameter in a neighbourhood of zero (see Section 2, where the reduction procedure is explained in detail).

Eigenvalues $\lambda$ of our infinite-dimensional Hamiltonian system with corresponding eigenvectors in the $k$ th Fourier mode ('mode $k$ eigenvalues') are associated with nonzero solutions of (1)-(4) whose $x$ - and $z$-dependence is described by $\mathrm{e}^{\mathrm{i} \nu k x} \mathrm{e}^{\lambda z}$. An elementary calculation shows that such eigenvalues satisfy

$$
\left(\alpha-\beta \sigma^{2}\right) \sigma \sin \sigma=\nu^{2} k^{2} \cos \sigma, \quad \sigma^{2}=\lambda^{2}-\nu^{2} k^{2}
$$

they are clearly geometrically double since the combination of periodicity and translation invariance of equations (1)-(4) in the $x$-direction generates an $O(2)$-symmetry. It follows from this formula that all eigenvalues are either real or purely imaginary and that for each $k$ there are either zero or two non-zero purely imaginary mode $k$ eigenvalues. Figure 1 shows how the number of purely imaginary eigenvalues changes at each of a countably infinite number of straight lines $C_{1}, C_{2}, \ldots$ in $(\beta, \alpha)$-parameter space; at each point of the line $C_{k}$ two real mode $k$ eigenvalues become purely imaginary by passing through zero in a non-semisimple resonance. We now make mode $k$ eigenvalues geometrically simple by exploiting the symmetry $(x, \rho(x, z), \phi(x, y, z)) \mapsto(-x, \rho(-x, z),-\phi(-x, y, z))$ of the hydrodynamic problem (1)-(4): 
we restrict attention to those solutions which are invariant with respect to this symmetry, so that their $\rho$ - and $\phi$-components are described by respectively cosine and sine series in $x$.

The line $C_{k}$ defined above consists of those positive values of $\alpha$ and $\beta$ which satisfy the equation

$$
\left(\alpha+\beta \nu^{2} k^{2}\right) \sinh \nu k=\nu k \cosh \nu k ;
$$

it connects the point $\left(\frac{1}{\nu k} \operatorname{coth} \nu k, 0\right)$ on the $\beta$-axis with the point $(0, \nu k \operatorname{coth} \nu k)$ on the $\alpha$-axis. Starting from the $\beta$-axis and moving left, we find that the line $C_{k}$ intersects each of the lines $C_{1}, \ldots, C_{k-1}, C_{k+1}, C_{k+2}, \ldots$ in turn before arriving at the $\alpha$-axis, and we denote the point of intersection of $C_{i}$ and $C_{j}, i>j$ by $P_{i, j}$ (see Figure 1). A Hamiltonian $0^{2}$-resonance therefore occurs at those points of $C_{1}$ to the right of $P_{1,2}$ and those points of $C_{j}$ between $P_{j-1, j}$ and $P_{j, j+1}$ for $j \geq 2$ : the purely imaginary spectrum consists of a geometrically simple zero eigenvalue which has a Jordan chain of length 2 . Taking reference values $\left(\beta_{0}, \alpha_{0}\right)$ as one of these points we obtain a two-dimensional centre manifold, the reduced Hamiltonian flow on which was discussed by Groves [6], who found that bifurcation of unipulse homoclinic orbits takes place to the right. In the present paper our interest lies however in homoclinic bifurcation associated with the Hamiltonian $0^{2} 0^{2}$-resonance at the codimension-two points $P_{j, j+1}, j=1,2, \ldots$; here the purely imaginary spectrum consists of a geometrically simple mode $k$ zero eigenvalue and a geometrically simple mode $(k+1)$ zero eigenvalue, each with a Jordan chain of length 2 , so that the zero eigenspace and generalised eigenspace are respectively two- and four-dimensional. Taking reference values $\left(\beta_{0}, \alpha_{0}\right)=P_{k, k+1}$ we obtain a four-dimensional centre manifold which captures the small-amplitude dynamics of the present water-wave problem in a full neighbourhood of $P_{k, k+1}$.

Notice that mode $i$ and mode $j$ eigenvalues can cross on the imaginary or real axes in a semisimple resonance. A straightforward calculation shows that a pair of mode $i$ and and a pair of mode $j$ purely imaginary eigenvalues with values $\pm s$ coincide at those points

$$
\begin{aligned}
\beta & =\frac{j^{2} \operatorname{coth} \gamma_{j}}{\left(j^{2}-i^{2}\right) \gamma_{j}}-\frac{i^{2} \operatorname{coth} \gamma_{i}}{\gamma_{j}\left(j^{2}-i^{2}\right)}, \\
\alpha & =\frac{i^{2} \gamma_{j}^{2} \operatorname{coth} \gamma_{i}}{\left(j^{2}-i^{2}\right) \gamma_{i}}-\frac{j^{2} \gamma_{i}^{2} \operatorname{coth} \gamma_{j}}{\left(j^{2}-i^{2}\right) \gamma_{j}}
\end{aligned}
$$

where

$$
\gamma_{i}^{2}=s^{2}+\nu^{2} i^{2}, \quad \gamma_{j}^{2}=s^{2}+\nu^{2} j^{2},
$$

for which the right-hand sides of (6), (7) are positive. These points constitute a single-branched curve $I_{i, j}$ in the $(\beta, \alpha)$-parameter plane which is parameterised by $s$. Similarly, we find that a pair of mode $i$ and and a pair of mode $j$ real eigenvalues with values $\pm \mathrm{i} \kappa$ coincide at those points

$$
\begin{aligned}
\beta & =\frac{i^{2} \cot \sigma_{i}}{\left(j^{2}-i^{2}\right) \sigma_{i}}-\frac{j^{2} \cot \sigma_{j}}{\left(j^{2}-i^{2}\right) \sigma_{j}}, \\
\alpha & =\frac{i^{2} \sigma_{j}^{2} \cot \sigma_{i}}{\left(j^{2}-i^{2}\right) \sigma_{i}}-\frac{j^{2} \sigma_{i}^{2} \cot \sigma_{j}}{\left(j^{2}-i^{2}\right) \sigma_{j}}
\end{aligned}
$$

where

$$
\sigma_{i}^{2}=\kappa^{2}-\nu^{2} i^{2}, \quad \sigma_{j}^{2}=\kappa^{2}-\nu^{2} j^{2},
$$

for which the right-hand sides of (8), (9) are positive. These points constitute a multi-branched curve $R_{i, j}$ in the $(\beta, \alpha)$-parameter plane which is parameterised by $\kappa$. Of particular interest here 


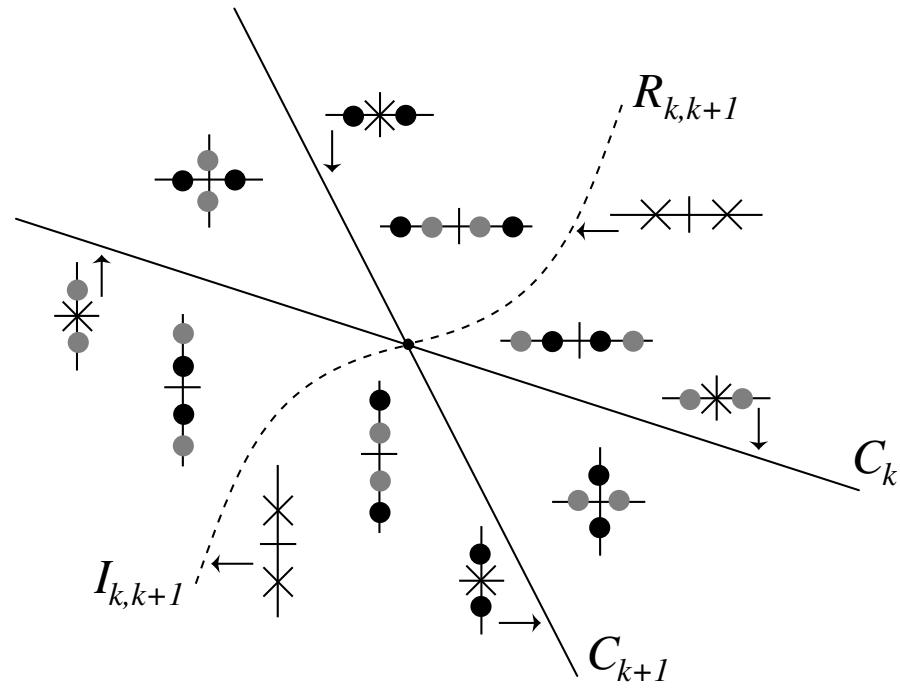

Figure 2: Behaviour of mode $k$ (black) and mode $k+1$ (grey) eigenvalues in a neighbourhood of the codimension-two point $P_{k, k+1}$. Semisimple and non-semisimple eigenvalue resonances take place on respectively the dashed and solid lines.

are the local parts of $I_{k, k+1}$ and $R_{k, k+1}$ near the points $P_{k, k+1}$, since we can access these curves using the centre-manifold reduction technique with reference values $\left(\beta_{0}, \alpha_{0}\right)=P_{k, k+1}$. The reduced Hamiltonian system is four-dimensional and captures the Hamiltonian $0^{2} 0^{2}$-resonance at the point $P_{k, k+1}$ itself, together with the Hamiltonian semisimple $1: 1$ resonance and the Hamiltonian real semisimple 1 : 1 resonance which occur on the local parts of respectively $I_{k, k+1}$ and $R_{k, k+1}$ near $P_{k, k+1}$ (see Figure 2). A general theory for the Hamiltonian semisimple 1 : 1 resonance has been developed by Cotter [3], and is in principle applicable to the local part of $I_{k, k+1}$ here; it consists of a bifurcation theory for periodic solutions. However in the present paper we focus upon homoclinic bifurcation and turn our attention to the Hamiltonian real semisimple $1: 1$ resonance associated with the local part of $R_{k, k+1}$. We therefore choose values of the bifurcation parameters $\epsilon_{1}, \epsilon_{2}$ in a fashion which enables us to access this curve effectively, namely by writing

$$
\epsilon_{1}=P_{k, k+1}^{\beta}+\hat{\beta}_{k, k+1} \mu^{2}, \quad \epsilon_{2}=P_{k, k+1}^{\alpha}+\left(\hat{\alpha}_{k, k+1}+\delta\right) \mu^{2},
$$

where $\hat{\beta}_{k, k+1}, \hat{\alpha}_{k, k+1}$ are the coefficients of $s^{2}$ in the Taylor expansion of the right-hand sides of (8), (9) and $\delta$ and $\mu$ are small positive numbers. The former parameter $\delta$ plays the role of a bifurcation parameter (varying $\delta$ through zero from above we cross the critical curve $R_{k, k+1}$ in parameter space from above), while the latter parameter $\mu$ indicates the distance from the point $P_{k, k+1}$.

The lines $C_{k}$ also play a central role in bifurcation theory for the two-dimensional travelling water waves. We have characterised points on the line $C_{k}$ in $(\beta, \alpha)$-parameter space as values of the physical parameters at which a mode $k$ zero eigenvalue exists. A mode $k$ zero eigenvalue arises when the linearised version of the travelling water-wave problem (1)-(4) admits a solution whose $x$ - and $z$-dependence is described by $\mathrm{e}^{\mathrm{i} \nu k x}$, and a solution of this form is clearly a periodic solution of the two-dimensional ( $z$-independent) linear travelling water-wave problem with 
frequency $k \nu$. A necessary condition for the bifurcation of nonlinear Stokes waves (nonlinear two-dimensional periodic waves) with this frequency from the uniform flow is therefore satisfied at points on this curve. This observation was taken up by Jones [14], who used a formulation of the two-dimensional travelling water-wave problem as an integral equation to analyse primary and secondary bifurcation phenomena for Stokes waves. Jones was particularly interested in the points $P_{i, j}$ at which $C_{i}$ intersects $C_{j}$. Here we have characterised these points as points at which mode $i$ and mode $j$ zero eigenvalues of our evolutionary system simultaneously exist, but they can also be understood as points associated with the simultaneous existence of the $i$ th and $j$ th harmonics of a fundamental periodic two-dimensional travelling wave ('Wilton ripples'). Observe that Stokes waves are equilibrium solutions of our evolutionary system and Jones's results can therefore in principle be recovered by examining steady-state bifurcations in the reduced equations on the centre manifold. This programme is however outside the scope of the present paper. Further remarks pertaining to the connection between our evolutionary system and the two-dimensional water-wave problem are given by Groves [6, §1.3].

\subsection{Homoclinic bifurcation}

Homoclinic bifurcation associated with the Hamiltonian real semisimple $1: 1$ resonance has recently been examined by Yew [24] and Yew, Sandstede \& Jones [25], who observed this phenomenon in the travelling-wave reduction of a system of coupled nonlinear Schrödinger equations which arises as a model in nonlinear optics. Their theory is an application of the homoclinic Lyapunov-Schmidt theory developed by Lin [18] and Sandstede [21, §3], which has also been successfully applied to other bifurcation scenarios, notably 'orbit-flip' bifurcations (see Sandstede, Jones \& Alexander [22]). Although the homoclinic Lyapunov-Schmidt theory is rather general, several major simplifications are afforded by the special structure of the system of equations treated in the present paper, in particular by the fact that we are considering a reversible Hamiltonian system with symmetries. A brief explanation of the theory in the form required here is presented in Section 4.1.

The essential ingredients of the homoclinic Lyapunov-Schmidt theory are the existence of one or more primary homoclinic orbits $h_{1}, \ldots, h_{m}$ at criticality and one or more discrete $\mathbb{Z}_{2^{-}}$ symmetries $T_{1}, \ldots, T_{n}$ of the system under discussion. The primary homoclinic orbits are unipulse solutions and are required to be transverse in the usual sense in Hamiltonian-systems theory: they correspond to intersections of the stable and unstable manifolds of the zero equilibrium which are transverse within the zero energy surface of the Hamiltonian function. As a consequence of their transversality, the primary homoclinic solutions persist for small, nonzero values of the bifurcation parameter, where they are denoted by $h_{1}^{\epsilon}, \ldots, h_{m}^{\epsilon}$. One then seeks multipulse solutions which resemble concatenations of an arbitrary number $N$ of unipulse solutions $u_{1}^{\epsilon}, \ldots, u_{N}^{\epsilon}$, each obtained by applying the reflectors $T_{1}, \ldots, T_{n}$ to the catalogue $h_{1}^{\epsilon}, \ldots, h_{m}^{\epsilon}$ of basic primary homoclinic orbits. The homoclinic Lyapunov-Schmidt theory consists of a procedure for reducing this question to a bifurcation equation for $N-1$ 'times of flight' of orbits close to the primary homoclinic solutions; the solvability of the bifurcation equation is addressed using asymptotic information from the 'tails' of the homoclinic orbits.

The basic travelling water-wave problem (1)-(4) has two $\mathbb{Z}_{2}$-symmetries, namely $z \mapsto-z$, which manifests itself in the fact that the Hamiltonian formulation of the problem is reversible, and $(x, \rho(x, z), \phi(x, y, z)) \mapsto(-x, \rho(-x, z),-\phi(-x, y, z))$, which generates a corres- 
ponding $\mathbb{Z}_{2}$-symmetry of the Hamiltonian formulation. It also has the continuous symmetry $x \mapsto x+a, a \in \mathbb{R}$, which likewise generates a continuous symmetry of the Hamiltonian formulation. These symmetries are inherited by the reduced Hamiltonian system at the points $P_{k, k+1}$, which are reversible and have a $\mathbb{Z}_{2}$ - and a continuous symmetry; the actions of the corresponding reverser $S$, reflector $R$ and translator $T_{a}$ are described in appropriate canonical coordinates in Section 3.1 (see in particular equations (29)-(31)). Restricting to solutions which are invariant with respect to the reflector $R$ (which correspond to solutions of the hydrodynamic equations (1)-(4) which are invariant with respect to the symmetry $(x, \rho(x, z), \phi(x, y, z)) \mapsto$ $(-x, \rho(-x, z),-\phi(-x, y, z))$, we obtain a two-degree-of-freedom Hamiltonian system with canonical coordinates $\left(q_{1}, q_{2}, p_{1}, p_{2}\right)$; the continuous symmetry $T_{a}$ is replaced at $P_{k, k+1}$ by the $\mathbb{Z}_{2}$-symmetry with reflector $T_{1}:\left(q_{1}, q_{2}, p_{1}, p_{2}\right) \mapsto\left(-q_{1}, q_{2},-p_{1}, p_{2}\right)$ when $k$ is odd and by the $\mathbb{Z}_{2}$-symmetry with reflector $T_{2}:\left(q_{1}, q_{2}, p_{1}, p_{2}\right) \mapsto\left(q_{1},-q_{2}, p_{1},-p_{2}\right)$ when $k$ is even (see Proposition 3.1 for a detailed explanation).

The reduced system of equations for $k=1$ is computed in Section 3.2. Examining first the flow in the invariant subspace Fix $T_{1}=\left\{\left(q_{1}, p_{1}\right)=(0,0)\right\}$, we find that it has the additional $\mathbb{Z}_{2^{-}}$ symmetry with reflector $U_{2}:\left(q_{2}, p_{2}\right) \mapsto\left(-q_{2},-p_{2}\right)$. The leading-order terms in the nonlinearity are cubic and the reduced equations admit a pair of unipulse transverse homoclinic orbits $u_{2}^{\mu, \delta}$, $U_{2} u_{2}^{\mu, \delta}$ for small values of the parameters $\mu$ and $\delta$. Tracing back the various changes of variable, we obtain the asymptotic formula

$$
\rho(x, z)=\frac{2 \sqrt{2} \mu}{\left(\gamma_{2} d\right)^{1 / 2}} \sinh 2 \nu \operatorname{sech} \mu z \cos 2 \nu x+O\left(\mu^{2}\right)
$$

for the corresponding water wave at $\delta=0$; the action of the reflector $U_{2}$ corresponds to the translation $x \mapsto x+\pi / 2 \nu$. Recall that our waves are $2 \pi / \nu$-periodic in $x$, so that the leadingorder term in the above expansion describes a wave associated with the second harmonic in the $x$-direction. In the complete four-dimensional phase space the leading-order terms in the nonlinearity are quadratic, and the reduced equations admit another unipulse transverse homoclinic orbit $u_{1,2}^{\mu, 0}$ at $\delta=0$ corresponding to the travelling water wave

$$
\rho(x, z)=\frac{6 \mu^{2}}{\left(2 \gamma_{1}\right)^{1 / 2} c} \sinh \nu \operatorname{sech}^{2}\left(\frac{\mu z}{2}\right) \cos \nu x+\frac{6 \mu^{2}}{2 \gamma_{2}^{1 / 2} c} \sinh 2 \nu \operatorname{sech}^{2}\left(\frac{\mu z}{2}\right) \cos 2 \nu x+O\left(\mu^{3}\right),
$$

which at leading order is associated with the first and second harmonics in the $x$-direction; a further homoclinic orbit is obtained using the reflector $T_{1}$, whose action corresponds to the translation $x \mapsto x+\pi / \nu$. (The constants $\gamma_{1}, \gamma_{2}$ and $c, d$ in the above discussion are given in equation (28) and Appendix A.)

The reduced equations for $k \geq 2$ are treated in Section 3.3. It follows from the combinatorics of the $k: k+1$ mode interaction that the leading-order terms in the nonlinearity are cubic and that both $\left\{\left(q_{1}, p_{1}\right)=(0,0)\right\}$ and $\left\{\left(q_{2}, p_{2}\right)=(0,0)\right\}$ are invariant subspaces under the flow generated by the Taylor expansion of the Hamiltonian vector field to every order; these subspaces are equipped with the $\mathbb{Z}_{2}$-symmetries with reflectors $U_{2}:\left(q_{2}, p_{2}\right) \mapsto\left(-q_{2},-p_{2}\right)$ and $U_{1}:\left(q_{1}, p_{1}\right) \mapsto\left(-q_{1},-p_{1}\right)$ respectively. (The invariance of $\left\{\left(q_{1}, p_{1}\right)=(0,0)\right\}$ for odd values of $k$ and $\left\{\left(q_{2}, p_{2}\right)=(0,0)\right\}$ for even values of $k$ is already assured by the fact that these subspaces correspond to Fix $T_{1}$ and Fix $T_{2}$.) We find that $\left\{\left(q_{2}, p_{2}\right)=(0,0)\right\}$ and $\left\{\left(q_{1}, p_{1}\right)=(0,0)\right\}$ 


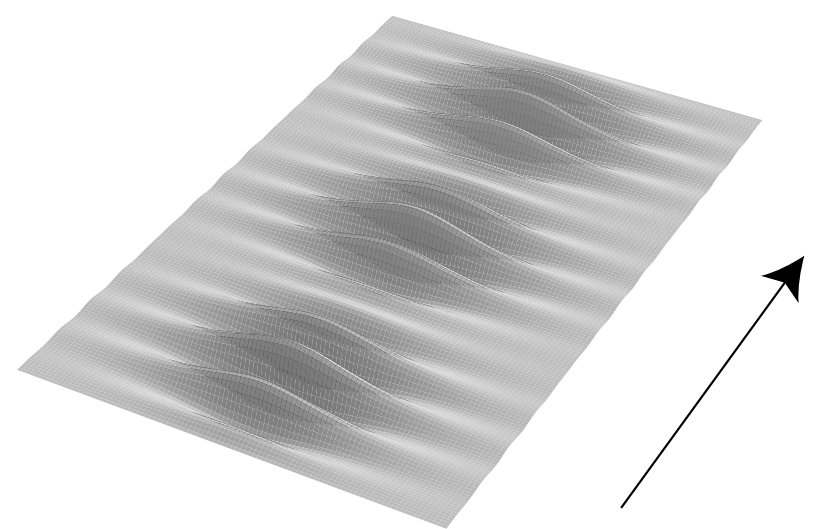

Figure 3: This periodic travelling wave is associated with two higher harmonics in the $x$ direction; it has a pulse-like profile in the z-direction. The arrow shows the direction of propagation.

each contain a pair of unipulse homoclinic orbits denoted by respectively $u_{k}^{\mu, \delta}, U_{1} u_{k}^{\mu, \delta}$ and $u_{k+1}^{\mu, \delta}$, $U_{2} u_{k+1}^{\mu, \delta}$. These orbits yield the travelling water waves given at $\delta=0$ by the asymptotic formulae

$$
\rho(x, z)=\frac{2 \sqrt{2} \mu}{\left(\gamma_{k} c_{1}\right)^{1 / 2}} \sinh k \nu \operatorname{sech} \mu z \cos k \nu x+O\left(\mu^{2}\right),
$$

which at leading order is associated with the $k$ th harmonic in the $x$-direction, and

$$
\rho(x, z)=\frac{2 \sqrt{2} \mu}{\left(\gamma_{k+1} c_{2}\right)^{1 / 2}} \sinh (k+1) \nu \operatorname{sech} \mu z \cos (k+1) \nu x+O\left(\mu^{2}\right),
$$

which at leading order is associated with the $(k+1)$ the harmonic in the $x$-direction; the actions of the reflectors $U_{1}, U_{2}$ correspond to respectively $x \mapsto x+\pi / k \nu$ and $x \mapsto x+\pi /(k+1) \nu$. In the complete four-dimensional phase space the reduced equations admit another unipulse transverse homoclinic orbit $u_{k, k+1}^{\mu, 0}$ at $\delta=0$ corresponding to the travelling water wave

$$
\begin{aligned}
\rho(x, z)=2 \mu & \left(\frac{2\left(c_{2}-c_{3}\right)}{\gamma_{k}\left(c_{1} c_{2}-c_{3}^{2}\right)}\right)^{1 / 2} \sinh k \nu \operatorname{sech} \mu z \cos k \nu x \\
& +2 \mu\left(\frac{2\left(c_{2}-c_{3}\right)}{\gamma_{k+1}\left(c_{1} c_{2}-c_{3}^{2}\right)}\right)^{1 / 2} \sinh (k+1) \nu \operatorname{sech} \mu z \cos (k+1) \nu x+O\left(\mu^{3}\right),
\end{aligned}
$$

which at leading order is associated with the $k$ th and $(k+1)$ th harmonics in $x$. A further homoclinic orbit is obtained using the reflector $T_{1}$ for odd values of $k$ or $T_{2}$ for even values of $k$; the action of the relevant reflector corresponds to the translation $x \mapsto x+\pi / \nu$. (The constants $\gamma_{k}$, $\gamma_{k+1}$ and $c_{1}, c_{2}, c_{3}$ are again given in equation (28) and Appendix A.)

The homoclinic Lyapunov-Schmidt method is applied to the reduced equations at $P_{k, k+1}$ in Section 4.2. A key requirement of the method as it relates to our problem is that the primary homoclinic orbits should have non-vanishing components in all four coordinates; for this reason we use the single primary homoclinic orbit $u_{k, k+1}^{\mu, \delta}$ and of course the single reflector $T_{1}$ for odd values of $k$ or $T_{2}$ for even values of $k$. Figure 3 shows a sketch of a water wave corresponding to 


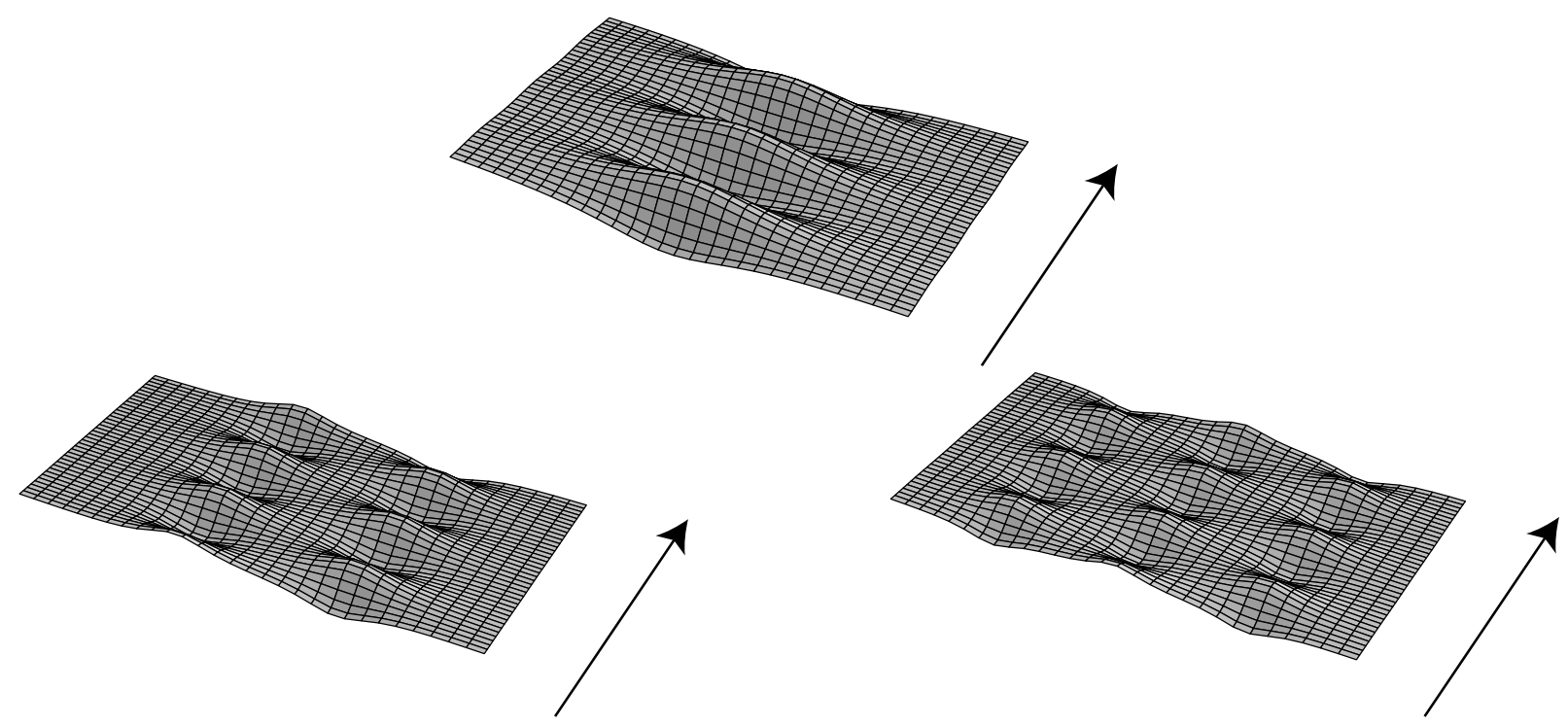

Figure 4: Sketches of uni- and multipulse travelling water waves near $P_{k, k+1}$; for clarity only the first harmonic is shown. Successive pulses in the z-direction are out of phase by one half of the period in the $x$-direction.

a typical unipulse homoclinic orbit of this kind, that is a water wave associated with two higher harmonics in the $x$-direction. The bifurcation result is the same for $k=1$ and $k \geq 2$ : multipulse homoclinic solutions with an arbitrary number of excursions away from the zero equilibrium are found to exist for $\delta>0$ (Theorem 4.5); the corresponding regions of $(\beta, \alpha)$-parameter space are marked in Figure 1. Our multipulse homoclinic orbits resemble a concatenation of $u_{k, k+1}^{\mu, \delta}$ and $T_{1} u_{k, k_{+}}^{\mu, \delta}$ or $T_{2} u_{k, k+1}^{\mu \delta}$ in a strictly alternating sequence, so that the corresponding travelling water waves are $2 \pi / \nu$-periodic in $x$ and have a large-scale structure consisting of a multipulse profile in $z$ whose successive pulses are out of phase by $\pi / \nu$ (see Figure 4). Schematic bifurcation diagrams for $k=1$ and $k \geq 2$ are shown in Figure 5 .

Our results complement those obtained by Buffoni, Groves \& Toland [2], who studied homoclinic bifurcation near a codimension-two point in parameter space for the two-dimensional travelling water-wave problem by means of a four-dimensional Hamiltonian centre-manifold reduction. In that paper the codimension-two point is associated with a Hamiltonian $0^{4}$-resonance (a geometrically simple zero eigenvalue with a Jordan chain of length 4), and local curves associated with a Hamiltonian non-semisimple 1 : 1 resonance and a Hamiltonian real non-semisimple $1: 1$ resonance (two geometrically simple respectively purely imaginary and real eigenvalues, each with a Jordan chain of length 2) emerge from the codimension-two point. A unipulse transverse homoclinic orbit is detected near the latter curve, and a family of multipulse homoclinic orbits are constructed in the neighbouring region where the eigenvalues are complex using a result of Devaney [5]. Eigenvalue resonances in Hamiltonian systems are generically non-semisimple, and the tails of the homoclinic orbits obtained by Devaney's construction decay exponentially to zero in an oscillatory fashion. By contrast the eigenvalue resonances in the present paper are semisimple and therefore non-generic; our homoclinic orbits decay exponentially to zero in a monotonic fashion since the eigenvalues of our reduced Hamiltonian system are real in the relevant parameter regime. 

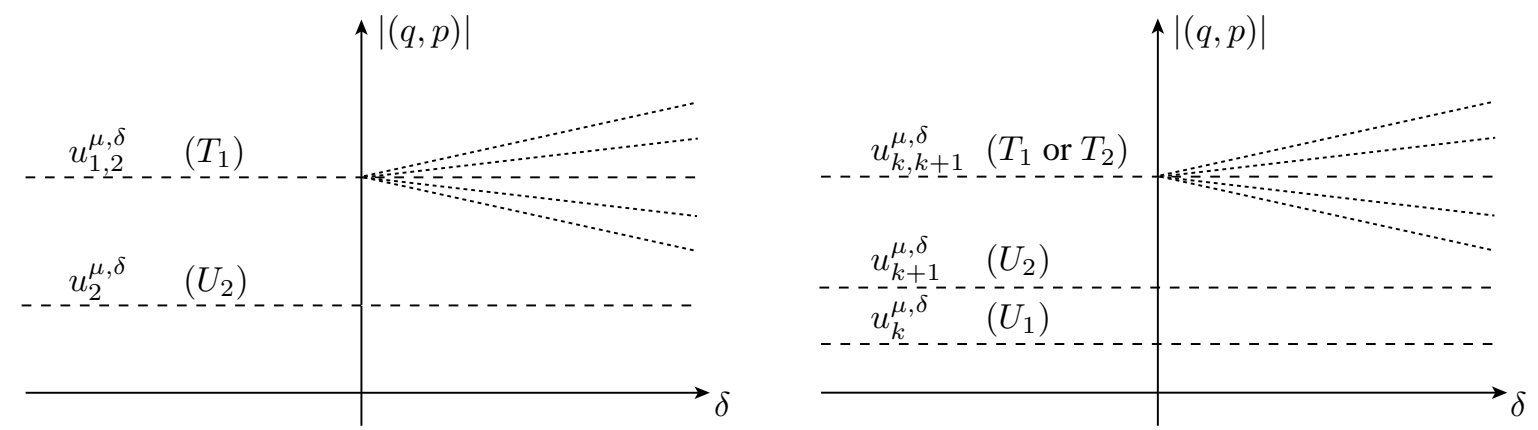

Figure 5: Schematic homoclinic bifurcation diagrams for the reduced Hamiltonian system for $\mu>0$ and $(a) k=1$ and $(b) k \geq 2$. Uni- and multipulse homoclinic solutions are depicted by respectively dashed and dotted lines; symbols in parentheses indicate reflectors which can be applied to obtain further unipulse homoclinic orbits.

Acknowledgements M.D.G. wishes to record his thanks for the warm hospitality shown by the Mathematisches Institut I, Universität Karlsruhe during an extended visit in which this paper was written. B.S. was partially supported by the NSF under grant DMS-0203854 and by an Alfred P. Sloan Research Fellowship.

\section{Hamiltonian centre-manifold reduction}

The cornerstone of our analysis is a formulation of the steady water-wave problem (1)-(4) as a Hamiltonian system in terms of the time-like variable $z$, where the waves are supposed to be periodic in $x$ with fixed frequency $\nu$. This matter has been addressed in detail by Groves [6], who used the canonical variables $\rho(x, z)$ and

$$
\Phi(x, y, z):=\phi(x, Y, z), \quad y=Y /(1+\rho)
$$

and their conjugate momenta

$$
\begin{aligned}
\omega(x, z) & :=-\int_{0}^{1}\left(\Phi_{z}-\frac{\Phi_{y} y \rho_{z}}{1+\rho}\right) \frac{y \Phi_{y}}{1+\rho} \mathrm{d} y+\frac{\beta \rho_{z}}{\sqrt{1+\rho_{x}^{2}+\rho_{z}^{2}}}, \\
\Psi(x, y, z) & :=\left(\Phi_{z}-\frac{\Phi_{y} y \rho_{z}}{1+\rho}\right)(1+\rho) .
\end{aligned}
$$

Here we confine ourselves to a brief description of this Hamiltonian spatial dynamics formulation and defer to the above reference for full details. We incorporate the frequency $\nu$ of the waves in the $x$-direction into our problem by introducing a new variable $x^{\prime}=\nu x$ and studying waves which are $2 \pi$-periodic in $x^{\prime}$; for notational simplicity we drop the prime in the following analysis.

The Hamiltonian system in question is defined upon the symplectic manifold $(M, \Omega)$, where $M$ is the function space

$$
X_{s}=H_{\mathrm{per}}^{s+1}(S) \times H_{\mathrm{per}}^{s}(S) \times H_{\mathrm{per}}^{s+1}(\Sigma) \times H_{\mathrm{per}}^{s}(\Sigma), \quad s \in(0,1 / 2)
$$


with

$$
\begin{aligned}
& H_{\mathrm{per}}^{s}(S)=\left\{u \in H_{\mathrm{loc}}^{t}(\mathbb{R}): u(x+2 \pi)=u(x), x \in \mathbb{R}\right\} \\
& H_{\mathrm{per}}^{s}(\Sigma)=\left\{u \in H_{\mathrm{loc}}^{t}((0,1) \times \mathbb{R}): u(x+2 \pi, y)=u(x, y), x \in \mathbb{R}, y \in(0,1)\right\}
\end{aligned}
$$

$S=(0,2 \pi), \Sigma=(0,1) \times(0,2 \pi)$ and the position-independent 2 -form $\Omega$ is given by

$$
\begin{aligned}
& \Omega\left(\left(\rho_{1}, \omega_{1}, \Phi_{1}, \Psi_{1}\right),\left(\rho_{2}, \omega_{2}, \Phi_{2}, \Psi_{2}\right)\right)= \\
& \int_{S}\left(\omega_{2} \rho_{1}-\rho_{2} \omega_{1}\right) \mathrm{d} z+\int_{\Sigma}\left(\Psi_{2} \Phi_{1}-\Phi_{2} \Psi_{1}\right) \mathrm{d} y \mathrm{~d} z
\end{aligned}
$$

(the canonical 2-form with respect to the $L^{2}(S) \times L^{2}(S) \times L^{2}(\Sigma) \times L^{2}(\Sigma)$-inner product). We proceed by fixing 'reference values' $\beta_{0}, \alpha_{0}$ of the physical parameters $\beta$ and $\alpha$ and perturbing around them with real bifurcation parameters $\epsilon_{1}, \epsilon_{2}$ which lie in neighbourhoods $\Lambda_{1}, \Lambda_{2}$ of the origin in $\mathbb{R}$ and choosing a neighbourhood $U$ of the origin in $X_{s+1}$ which is small enough so that

$$
\rho(x)>-\frac{1}{2}>-1, \quad\left|\epsilon_{2}\right|<\frac{1}{4} \beta_{0}, \quad|W(x)|<\frac{\beta_{0}}{2}<\beta_{0}+\epsilon_{2}
$$

for all $x \in[0,2 \pi]$. The Hamiltonian function $H^{\epsilon} \in C^{\infty}(U, \mathbb{R})$ is defined by the formula

$$
\begin{aligned}
& H^{\epsilon}(\rho, \omega, \Phi, \Psi) \\
& =\int_{\Sigma}\left\{(1+\rho) \Phi_{x}-\nu y \rho_{x} \Phi_{y}+\frac{\Psi^{2}-\Phi_{y}^{2}}{2(1+\rho)}-\frac{\nu^{2}(1+\rho)}{2}\left(\Phi_{x}-\frac{y \rho_{x} \Phi_{y}}{1+\rho}\right)^{2}\right\} \mathrm{d} y \mathrm{~d} x \\
& \quad+\int_{S}\left\{-\frac{1}{2}\left(\alpha_{0}+\epsilon_{1}\right) \rho^{2}+\beta_{0}+\epsilon_{2}-\left(\left(\beta_{0}+\epsilon_{2}\right)^{2}-W^{2}\right)^{\frac{1}{2}}\left(1+\nu^{2} \rho_{x}^{2}\right)^{\frac{1}{2}}\right\} \mathrm{d} x,
\end{aligned}
$$

where

$$
W=\omega+\frac{1}{1+\rho} \int_{0}^{1} y \Phi_{y} \Psi \mathrm{d} y
$$

and we note that $U$ is a manifold domain of $M$.

In order to compute the Hamiltonian vector field $v_{H}^{\epsilon}$ corresponding to the Hamiltonian system $\left(M, \Omega, H^{\epsilon}\right)$, recall that the point $m \in U$ belongs to $\mathcal{D}\left(v_{H}\right)$ with $\left.v_{H}\right|_{m}=\left.\bar{v}\right|_{m}$ if and only if

$$
\left.\Omega\right|_{m}\left(\left.\bar{v}\right|_{m},\left.v_{1}\right|_{m}\right)=\left.\mathbf{d} H\right|_{m}\left(\left.v_{1}\right|_{m}\right)
$$

for all tangent vectors $\left.\left.v_{1}\right|_{m} \in T M\right|_{m}$, where $\left.\mathbf{d} H\right|_{m}$ is understood as an element of $\left.T^{*} M\right|_{m} \cong X_{s}^{*}$ (an inspection of the formula for $\left.\mathbf{d} H\right|_{m}, m \in U$, which is a priori defined as an element of $\left.T^{*} U\right|_{m} \cong X_{s+1}^{*}$ shows that it admits a natural extension which lies in $\left.\left.T^{*} M\right|_{m} \cong X_{s}^{*}\right)$. Using this criterion, one finds that $\mathcal{D}\left(v_{H}\right)$ is the set of functions $(\rho, \omega, \Phi, \Psi) \in U$ that satisfy the natural boundary conditions

$$
\begin{array}{ll}
\Phi_{y}=0 & \text { on } y=0, \\
\nu \rho_{x}+\frac{\Phi_{y}}{\rho+1}=\nu^{2} \rho_{x}\left(\Phi_{x}-\frac{\rho_{x} \Phi_{y}}{\rho+1}\right)+\frac{W \Psi}{\rho+1}\left(\frac{1+\nu^{2} \rho_{x}^{2}}{\left(\beta_{0}+\epsilon_{2}\right)^{2}-W^{2}}\right)^{\frac{1}{2}} & \text { on } y=1
\end{array}
$$


and that Hamilton's equations

$$
u_{z}=v_{H}(u)
$$

are given explicitly by

$$
\begin{aligned}
\rho_{z}= & W\left(\frac{1+\nu^{2} \rho_{x}^{2}}{\left(\beta_{0}+\epsilon_{2}\right)^{2}-W^{2}}\right)^{\frac{1}{2}} \\
\omega_{z}= & \frac{W}{(\rho+1)^{2}}\left(\frac{1+\nu^{2} \rho_{x}^{2}}{\left(\beta_{0}+\epsilon_{2}\right)^{2}-W^{2}}\right)^{\frac{1}{2}} \int_{0}^{1} y \Phi_{y} \Psi \mathrm{d} y-\nu^{2}\left[\rho_{x}\left(\frac{\left(\beta_{0}+\epsilon_{2}\right)^{2}-W^{2}}{1+\nu^{2} \rho_{x}^{2}}\right)^{\frac{1}{2}}\right]_{x} \\
& +\int_{0}^{1}\left\{\frac{\Psi^{2}-\Phi_{y}^{2}}{2(\rho+1)^{2}}+\frac{1}{2} \nu^{2}\left(\Phi_{x}-\frac{y \rho_{x} \Phi_{y}}{\rho+1}\right)^{2}+\nu^{2}\left[\left(\Phi_{x}-\frac{y \rho_{x} \Phi_{y}}{\rho+1}\right) y \Phi_{y}\right]_{x}\right. \\
\Phi_{z}= & \frac{\Psi}{(\rho+1)}+\frac{W y \Phi_{y}}{\rho+1}\left(\frac{1+\nu^{2} \rho_{x}^{2}}{\left(\beta_{0}+\epsilon_{2}\right)^{2}-W^{2}}\right)^{\frac{1}{2}}, \\
\Psi_{z}= & -\frac{y \rho_{x} \Phi_{y}}{\rho+1}-\nu^{2}\left[(\rho+1)\left(\Phi_{x}-\frac{y \rho_{x} \Phi_{y}}{\rho+1}\right)\right]_{x}+\nu^{2}\left[\left(\Phi_{x}-\frac{y \rho_{x} \Phi_{y}}{\rho+1}\right) y \rho_{x}\right]_{y} \\
& +\frac{W(y \Psi)_{y}}{\rho+1}\left(\frac{1+\nu^{2} \rho_{x}^{2}}{\left(\beta_{0}+\epsilon_{2}\right)^{2}-W^{2}}\right)^{\frac{1}{2}} \cdot(1)
\end{aligned}
$$

The results presented in Lemma 1 of Ref. [6] show that the vector field defined by the righthand sides of (17)-(20) defines a smooth function $U \rightarrow X_{s}$ and that the boundary conditions (15), (16) are also well-defined. Equations (17)-(20) therefore constitute an infinite-dimensional, quasilinear evolutionary system which is accompanied by the nonlinear boundary conditions (15), (16).

The Hamiltonian system $\left(M, \Omega, H^{\epsilon}\right)$ has the conserved quantities

$$
H^{\epsilon}(\rho, \omega, \Phi, \Psi), \quad \int_{S} \omega \rho_{x} \mathrm{~d} x+\int_{\Sigma} \Psi \Phi_{x} \mathrm{~d} y \mathrm{~d} x, \quad \int_{\Sigma} \Psi \mathrm{d} y \mathrm{~d} x
$$

which are associated with continuous symmetries, namely the invariance of Hamilton's equations under translations in $z, x$ and $\Phi$. Hamilton's equations also inherit the discrete symmetries of the hydrodynamic problem (1)-(4). They are invariant under the reflection $R: X_{s} \rightarrow X_{s}$ given by

$$
R(\rho(x), \omega(x), \Phi(x), \Psi(x))=(\rho(-x), \omega(-x),-\Phi(-x),-\Psi(-x))
$$

and are reversible; the reverser $S: X_{s} \rightarrow X_{s}$ is defined by

$$
S(\rho, \omega, \Phi, \Psi)=(\rho,-\omega, \Phi,-\Psi) .
$$

The next step is a centre-manifold reduction theorem for the Hamiltonian system $\left(M, \Omega, H^{\epsilon}\right)$ which states that it is locally equivalent to a finite-dimensional Hamiltonian system. In order to 
formulate the requisite result it is necessary to introduce the operator $L_{s}: \mathcal{D}\left(L_{s}\right) \subset X_{s} \rightarrow X_{s}$ given by

$$
L_{s}\left(\begin{array}{c}
\rho \\
\omega \\
\Phi \\
\Psi
\end{array}\right)=\left(\begin{array}{c}
\frac{1}{\beta_{0}} \omega \\
-\left.\nu \Phi_{x}\right|_{y=1}+\alpha_{0} \rho-\beta_{0} \nu^{2} \rho_{x x} \\
\Psi \\
-\nu^{2} \Phi_{x x}-\Phi_{y y}
\end{array}\right) ;
$$

its domain $\mathcal{D}\left(L_{s}\right)$ is the subspace of elements of $X_{s+1}$ that satisfy the boundary conditions

$$
\begin{array}{ll}
\Phi_{y}=0 & \text { on } y=0, \\
\Phi_{y}+\nu \rho_{x}=0 & \text { on } y=1 .
\end{array}
$$

(The operator $L_{s}$ is the formal linearisation of $v_{H}^{\epsilon}$ at 0 .) The following result concerning the spectrum of $L_{s}$ is obtained by a series of straightforward explicit calculations.

Lemma 2.1 The operator $L_{s}: \mathcal{D}\left(L_{s}\right) \subset X_{s} \rightarrow X_{s}$ is closed and densely defined. Its spectrum consists entirely of isolated eigenvalues of finite algebraic multiplicity and $\sigma_{\mathrm{c}}\left(L_{s}\right):=\sigma\left(L_{s}\right) \cap \mathrm{i} \mathbb{R}$ is a finite set. A complex number $\lambda$ is a mode $k$ eigenvalue of $L_{s}$ if and only if

$$
\left(\alpha_{0}-\beta_{0} \sigma^{2}\right) \sigma \sin \sigma=\nu^{2} k^{2} \cos \sigma, \quad \sigma^{2}=\lambda^{2}-\nu^{2} k^{2} .
$$

Lemma 2.1 enables us to apply the spectral theory of Kato [15] to define the finite-dimensional centre subspace $X_{s}^{\mathrm{c}}:=P\left[X_{s}\right]$ of $L_{s}$ using the spectral projection $P: X_{s} \rightarrow X_{s}$ given by

$$
P x=\frac{1}{2 \pi \mathrm{i}} \int_{C}\left(L_{s}-\lambda I\right)^{-1} x \mathrm{~d} \lambda,
$$

in which $C$ is a closed curve in the resolvent set of $L_{s}$ which contains $\sigma_{\mathrm{c}}\left(L_{s}\right)$ and no other point of $\sigma\left(L_{s}\right)$. We are now in a position to state our principal reduction result which asserts that $\left(M, \Omega, H^{\epsilon}\right)$ is locally equivalent to a Hamiltonian system with a finite number of degrees of freedom. The theorem is proved by constructing a change of variable which transforms the evolutionary system (15)-(20) into an equivalent system with linear boundary conditions and applying Mielke's centre-manifold reduction result for quasilinear systems $[19,20]$ to the transformed system. Full details are given by Groves [6, §3].

Theorem 2.2 For each $n \geq 2$ there exist neighbourhoods $\tilde{\Lambda} \subset \Lambda, \tilde{U}_{1} \subset X_{s}^{\mathrm{c}}$ and $\tilde{U} \subset X_{s+1}$ of 0 and a reduction function $h(\cdot, \epsilon) \in C_{\mathrm{b}, \mathrm{u}}^{n}\left(\tilde{U}_{1}, \tilde{U}\right)$ which depends smoothly upon $\epsilon \in \tilde{\Lambda}_{1}$, satisfies $h(0,0)=0, \mathrm{~d}_{1} h[0,0]=0$ and has the following properties. The graph

$$
M_{\mathrm{c}}^{\epsilon}=\left\{u_{1}+h\left(u_{1}, \epsilon\right): u_{1} \in \tilde{U}_{1}\right\}
$$

is contained in $\mathcal{D}\left(v_{H}^{\epsilon}\right)$ and defines a centre manifold for the Hamiltonian system $\left(M, \Omega, H^{\epsilon}\right)$, so that

(1) $M_{C}^{\epsilon}$ is a locally invariant manifold of Hamilton's equations for $\left(M, \Omega, H^{\epsilon}\right)$ : through every point in $M_{C}^{\epsilon}$ there passes a unique solution of Hamilton's equations for $\left(M, \Omega, H^{\epsilon}\right)$ that remains on $M_{C}^{\epsilon}$ as long as it remains in $\tilde{U}$; 
(2) Every small bounded solution $u(z), z \in \mathbb{R}$ of Hamilton's equations for $\left(M, \Omega, H^{\epsilon}\right)$ that satisfies $u(z) \in \tilde{U}$ lies completely in $M_{\mathrm{c}}^{\epsilon}$;

(3) $M_{C}^{\epsilon}$ is a symplectic submanifold of $M$ and the flow determined by the Hamiltonian system $\left(M_{\mathrm{c}}^{\epsilon}, \tilde{\Omega}, \tilde{H}^{\epsilon}\right)$, where the tilde denotes restriction to $M_{\mathrm{c}}^{\epsilon}$, coincides with the flow on $M_{\mathrm{c}}^{\epsilon}$ determined by $\left(M, \Omega, H^{\epsilon}\right)$;

(4) Every solution $\tilde{u}:(a, b) \rightarrow \tilde{U}_{1}$ of Hamilton's equations for $\left(M_{\mathrm{c}}^{\epsilon}, \tilde{\Omega}, \tilde{H}^{\epsilon}\right)$ generates a solution of Hamilton's equations for $\left(M, \Omega, H^{\epsilon}\right)$.

\section{The reduced system near the codimension-two points}

\subsection{Coordinates for the centre manifold}

The reduction theory presented in Section 2 above shows that, for each fixed parameter value $\left(\beta_{0}, \alpha_{0}\right)$, the travelling water-wave problem is locally equivalent in parameter- and solution-space to the finite-dimensional Hamiltonian system $\left(M_{\mathrm{c}}^{\epsilon}, \tilde{\Omega}^{\epsilon}, \tilde{H}^{\epsilon}\right)$. We now introduce a convenient coordinate system in which to study this reduced Hamiltonian system. The centre manifold

$$
M_{\mathrm{c}}^{\epsilon}=\left\{u_{1}+h\left(u_{1}, \epsilon\right): u_{1} \in \tilde{U}_{1}\right\}
$$

is modelled upon the single coordinate chart $\tilde{U}_{1}$, which is an open subset of the finite-dimensional vector space $X_{s}^{\mathrm{c}}$, and in this coordinate system the reduced Hamiltonian function $\tilde{H}^{\epsilon}$ and reduced 2 -form $\tilde{\Omega}^{\epsilon}$ are given by

$$
\begin{aligned}
\tilde{H}^{\epsilon}\left(u_{1}\right) & =H^{\epsilon}\left(u_{1}+h\left(u_{1}, \epsilon\right)\right), \\
\left.\tilde{\Omega}^{\epsilon}\right|_{u_{1}}\left(v^{1}, v^{2}\right) & =\left.\Omega\right|_{u_{1}+h\left(u_{1}, \epsilon\right)}\left(v^{1}+\mathrm{d}_{1} h\left[u_{1}, \epsilon\right]\left(v^{1}\right), v^{2}+\mathrm{d}_{1} h\left[u_{1}, \epsilon\right]\left(v^{2}\right)\right) \\
& =\Omega\left(v^{1}, v^{2}\right)+O\left(\left|\left(\epsilon, u_{1}\right)\right|\right) .
\end{aligned}
$$

Using these formulae as a starting point, we construct a coordinate system for $X_{s}^{\mathrm{c}}$ with respect to which $\tilde{\Omega}$ is the canonical symplectic 2 -form $\Upsilon$, focusing upon the situation in which $\left(\beta_{0}, \alpha_{0}\right)$ is one of the codimension-two points $P_{k, k+1}$. We therefore write

$$
\beta=P_{k, k+1}^{\beta}+\epsilon_{1}, \quad \alpha=P_{k, k+1}^{\alpha}+\epsilon_{2}
$$

and observe that the reduced system $\left(M_{\mathrm{c}}^{\epsilon}, \tilde{\Omega}^{\epsilon}, \tilde{H}^{\epsilon}\right)$ captures the small-amplitude dynamics of the travelling water-wave problem for values of $\left(\epsilon_{1}, \epsilon_{2}\right)$ in a neighbourhood $\tilde{\Lambda}$ of the origin in $\mathbb{R}^{2}$.

The first step is to choose a basis for $X_{s}^{\mathrm{c}}$ consisting of generalised eigenvectors of $L_{s}$ which is symplectic with respect to $\Omega$. Observe that $\lambda=0, k=0$ is always a solution of (26), so that there is always a mode 0 zero eigenvector; this eigenvector has a Jordan chain of length 2. Choosing

$$
e=\frac{1}{\sqrt{2 \pi}}\left(\begin{array}{c}
0 \\
0 \\
1 \\
0
\end{array}\right), \quad f=\frac{1}{\sqrt{2 \pi}}\left(\begin{array}{l}
0 \\
0 \\
0 \\
1
\end{array}\right),
$$


one finds that $L_{s} e=0, L_{s} f=e$ and $\Omega(e, f)=1$. There are also geometrically double mode $k$ and mode $k+1$ zero eigenvalues, and each eigenvector has a Jordan chain of length 2 . The vectors

$$
\begin{gathered}
e_{k}^{+}=\frac{1}{\sqrt{\gamma_{k}}}\left(\begin{array}{c}
2 \sinh (\nu k) \cos (k x) \\
0 \\
2 \cosh (\nu k) \sin (k x) \\
0
\end{array}\right), \quad e_{k}^{-}=\frac{1}{\sqrt{\gamma_{k}}}\left(\begin{array}{c}
-2 \sinh (\nu k) \sin (k x) \\
0 \\
2 \cosh (\nu k) \cos (k x) \\
0
\end{array}\right), \\
f_{k}^{+}=\frac{1}{\sqrt{\gamma_{k}}}\left(\begin{array}{c}
0 \\
2 \beta_{0} \sinh (\nu k) \cos (k x) \\
0 \\
2 \cosh (\nu k) \sin (k x)
\end{array}\right), \quad f_{k}^{-}=\frac{1}{\sqrt{\gamma_{k}}}\left(\begin{array}{c}
0 \\
-2 \beta_{0} \sinh (\nu k) \sin (k x) \\
0 \\
2 \cosh (\nu k) \cos (k x)
\end{array}\right),
\end{gathered}
$$

where

$$
\gamma_{k}=2 \pi+4 \pi \beta_{0} \sinh ^{2}(\nu k)+\frac{\pi}{\nu k} \sinh (2 \nu k),
$$

satisfy $L e_{k}^{ \pm}=0, L f_{k}^{ \pm}=e_{ \pm}^{0, k}$; moreover $e_{k}^{+}, f_{k}^{+}$are symmetric with respect to the reflection $R$, $e_{k}^{-}, f_{k}^{-}$is antisymmetric with respect to this reflection, $\Omega\left(e_{k}^{+}, f_{k}^{+}\right)=1, \Omega\left(e_{k}^{-}, f_{k}^{-}\right)=1$ and the symplectic product of any other combination is zero. The eigenvectors $e_{k+1}^{+}, e_{k+1}^{-}$and generalised eigenvectors $f_{k+1}^{+}, f_{k+1}^{-}$corresponding to the mode $k+1$ zero eigenvalue are defined in the same fashion. We conclude that the generalised eigenspace corresponding to the zero eigenvalue is ten-dimensional and has the symplectic basis $\left\{e, e_{k}^{+}, e_{k}^{-}, e_{k+1}^{+}, e_{k+1}^{-}, f, f_{k}^{+}, f_{k}^{-}, f_{k+1}^{+}, f_{k+1}^{-}\right\}$; since there are no further purely imaginary eigenvalues, the same is true of the centre space $X_{s}^{\mathrm{c}}$. The coordinates $q, q_{k}^{+}, q_{k}^{-}, q_{k+1}^{+}, q_{k+1}^{-}, p, p_{k}^{+}, p_{k}^{-}, p_{k+1}^{+}, p_{k+1}^{-}$in the $e, e_{k}^{+}, e_{k}^{-}, e_{k+1}^{+}, e_{k+1}^{-}, f, f_{k}^{+}, f_{k}^{-}$, $f_{k+1}^{+}, f_{k+1}^{-}$directions are canonical coordinates for $X_{s}^{\mathrm{c}}$ with respect to $\Omega$ and the actions of the reflector $R$ defined by (21), the reverser $S$ defined by (22) and the translator $T_{a}: x \mapsto x+a$, $a \in \mathbb{R}$ on this generalised eigenspace are given by

$$
\begin{aligned}
& R\left(q, q_{k}^{+}, q_{k}^{-}, q_{k+1}^{+}, q_{k+1}^{-}, p, p_{k}^{+}, p_{k}^{-}, p_{k+1}^{+}, p_{k+1}^{-}\right) \\
& \quad=\left(q, q_{k}^{+},-q_{k}^{-}, q_{k+1}^{+},-q_{k+1}^{-}, p, p_{k}^{+},-p_{k}^{-}, p_{k+1}^{+},-p_{k+1}^{-}\right), \\
& \quad S\left(q, q_{k}^{+}, q_{k}^{-}, q_{k+1}^{+}, q_{k+1}^{-}, p, p_{k}^{+}, p_{k}^{-}, p_{k+1}^{+}, p_{k+1}^{-}\right) \\
& \quad=\left(q, q_{k}^{+}, q_{k}^{-}, q_{k+1}^{+}, q_{k+1}^{-},-p,-p_{k}^{+},-p_{k}^{-},-p_{k+1}^{+},-p_{k+1}^{-}\right), \\
& \quad T_{a}\left(q, q_{k}^{+}, q_{k}^{-}, q_{k+1}^{+}, q_{k+1}^{-}, p, p_{k}^{+}, p_{k}^{-}, p_{k+1}^{+}, p_{k+1}^{-}\right) \\
& \quad=\left(q, \mathcal{R}_{k a}\left(q_{k}^{+}, q_{k}^{-}\right), \mathcal{R}_{(k+1) a}\left(q_{k+1}^{+}, q_{k+1}^{-}\right), p, \mathcal{R}_{k a}\left(p_{k}^{+}, p_{k}^{-}\right), \mathcal{R}_{(k+1) a}\left(p_{k+1}^{+}, p_{k+1}^{-}\right)\right),
\end{aligned}
$$

where $\mathcal{R}_{\theta}$ is the $2 \times 2$ matrix representing a rotation through the angle $\theta$.

Writing

$$
u_{1}=q e+q_{k}^{+} e_{k}^{+}+q_{k}^{-} e_{k}^{-}+q_{k+1}^{+} e_{k+1}^{+}+q_{k+1}^{-} e_{k+1}^{-}+p f+p_{k}^{+} f_{k}^{+}+p_{k}^{-} f_{k}^{-}+p_{k+1}^{+} f_{k+1}^{+}+p_{k+1}^{-} f_{k+1}^{-}
$$

and using the formula (27) for the reduced 2 -form $\tilde{\Omega}^{\epsilon}$, we find that

$$
\left.\left.\tilde{\Omega}^{\epsilon}\right|_{u_{1}} v_{1}, v_{2}\right)=\Upsilon\left(v_{1}, v_{2}\right)+O\left(\left|\left(u_{1}, \epsilon\right)\right|\left|v_{1}\right|\left|v_{2}\right|\right),
$$

where $\Upsilon$ is the canonical symplectic 2 -form on $\mathbb{R}^{10}$, so that

$$
\Upsilon\left((\cdot)_{1},(\cdot)_{2}\right)=\left\langle K(\cdot)_{1},(\cdot)_{2}\right\rangle, \quad K=\left(\begin{array}{cc}
0 & -I \\
I & 0
\end{array}\right),
$$


in which $I$ is the $5 \times 5$ identity matrix and $\langle\cdot, \cdot\rangle$ is the $\mathbb{R}^{10}$-inner product. Finally, we apply a parameter-dependent version of Darboux's theorem which asserts that the 2 -form $\tilde{\Omega}^{\epsilon}$ can be transformed into $\Upsilon$ by a near-identity Darboux change of coordinates (cf. Buffoni \& Groves [1, Theorem 4]). Using the formula

$$
\tilde{H}^{\epsilon}\left(u_{1}\right)=H^{\epsilon}\left(u_{1}+h\left(u_{1}, \epsilon\right)\right)
$$

the estimate

$$
h\left(u_{1}, \epsilon\right)=O\left(\left|u_{1}\right|\left|\left(u_{1}, \epsilon\right)\right|\right)
$$

and the choice of symplectic basis for $X_{s}^{\mathrm{c}}$, we find that the leading-order terms in the quadratic part $\tilde{H}_{\mathrm{L}}^{\epsilon}$ of the Hamiltonian are given in this coordinate system by

$$
\tilde{H}_{\mathrm{L}}^{0}\left(u_{1}\right)=\frac{1}{2}\left(p_{k}^{+}\right)^{2}+\frac{1}{2}\left(p_{k}^{-}\right)^{2}+\frac{1}{2}\left(p_{k+1}^{+}\right)^{2}+\frac{1}{2}\left(p_{k+1}^{-}\right)^{2}
$$

for later use we denote the 'nonlinear' part of the Hamiltonian by $\tilde{H}_{\mathrm{NL}}^{\epsilon}$.

The centre-manifold reduction procedure preserves reversibility and symmetries, and it is also possible to select the Darboux transformation to preserve such characteristics of the original equations (Mielke [20]). Note in particular that Hamilton's equations are reversible and invariant under the $\mathbb{Z}_{2}$-symmetry $R$ and the continuous symmetry $T_{a}$ (the actions of the reverser, reflector and translator are correctly described by (29)-(31)). They therefore have $O(2)$ symmetry and Fix $\mathrm{R}$ is an invariant subspace. The variable $q$ is in fact cyclic, so that its conjugate $p$ is a conserved quantity (which will be set to zero in the following analysis), and the dimension of the reduced system of equations can always be reduced by two. A further reduction of order can be achieved by restricting to the invariant subspace Fix $R=\left\{q_{k}^{-}=q_{k+1}^{-}=p_{k}^{-}=p_{k+1}^{-}=0\right\}$. This procedure breaks the $O(2)$ symmetry of the reduced equations, for which $T_{a}$ is no longer a symmetry for arbitrary values of $a$.

Proposition 3.1 In the invariant subspace Fix $R$, the reduced system of equations has the following discrete symmetries.

(1) For odd values of $k$ : the $\mathbb{Z}_{2}$-symmetry

$$
T_{1}:\left(q_{k}^{+}, q_{k+1}^{+}, p_{k}^{+}, p_{k+1}^{+}\right) \mapsto\left(-q_{k}^{+}, q_{k+1}^{+},-p_{k}^{+}, p_{k+1}^{+}\right)
$$

and the $\mathbb{Z}_{2}$-symmetry

$$
U_{2}:\left(q_{k+1}^{+}, p_{k+1}^{+}\right) \mapsto\left(-q_{k+1}^{+},-p_{k+1}^{+}\right)
$$

in the invariant subspace Fix $T_{1}$.

(2) For even values of $k$ : the $\mathbb{Z}_{2}$-symmetry

$$
T_{2}:\left(q_{k}^{+}, q_{k+1}^{+}, p_{k}^{+}, p_{k+1}^{+}\right) \mapsto\left(q_{k}^{+},-q_{k+1}^{+}, p_{k}^{+},-p_{k+1}^{+}\right)
$$

and the $\mathbb{Z}_{2}$-symmetry

$$
U_{1}:\left(q_{k}^{+}, p_{k}^{+}\right) \mapsto\left(-q_{k}^{+},-p_{k}^{+}\right)
$$

in the invariant subspace Fix $T_{2}$. 
Proof Observe that $T_{a}$ remains a symmetry of the reduced system provided that $T_{a}[$ Fix $R]=$ Fix $R$. The calculation

$$
\begin{aligned}
T_{a}\left(q, q_{k}^{+}, 0, q_{k+1}^{+}, 0, p, p_{k}^{+}, 0, p_{k+1}^{+}, 0\right) \\
=\left(q, \mathcal{R}_{k a}\left(q_{k}^{+}, 0\right), \mathcal{R}_{(k+1) a}\left(q_{k+1}^{+}, 0\right), p, \mathcal{R}_{k a}\left(p_{k}^{+}, 0\right), \mathcal{R}_{(k+1) a}\left(p_{k+1}^{+}, 0\right)\right) \\
=\quad\left(q, \cos (k a) q_{k}^{+}, \sin (k a) q_{k}^{+}, \cos ((k+1) a) q_{k+1}^{+}, \sin ((k+1) a) q_{k+1}^{+},\right. \\
\left.\quad p, \cos (k a) p_{k}^{+}, \sin (k a) p_{k}^{+}, \cos ((k+1) a) p_{k+1}^{+}, \sin ((k+1) a) p_{k+1}^{+}\right),
\end{aligned}
$$

shows that $T_{a}[$ Fix $R]=$ Fix $R$ if and only if $k a=n_{1} \pi$ and $(k+1) a=n_{2} \pi$ for some positive integers $n_{1}, n_{2}$. It follows from the relation $k a=n_{1} \pi$ that $(k+1) a=(k+1) n_{1} \pi / k$ and therefore that $n_{2}=(k+1) n_{1} / k$. The quantity $(k+1) / k$ is an integer if and only if $k=1$, in which case it has the value 2 , so that $n_{2}=2 n_{1}$, and one finds that $T_{a}=T$ when $n_{1}$ is odd and $T_{a}$ is the identity when $n_{1}$ is even. For $k \geq 2$ the requirement that $n_{2}$ should be an integer implies that $n_{1}=m k$ for some positive integer $m$, whence $n_{2}=m(k+1)$, and one finds that $T_{a}$ is the identity when $m$ is even, that $T_{a}=T_{1}$ when $m$ and $k$ are odd and that $T_{a}=T_{2}$ when $m$ is odd and $k$ is even.

Similarly, for odd values of $k$ the mapping $T_{a}$ remains a symmetry of the reduced system in its invariant subspace Fix $R \cap$ Fix $T_{1}$ provided that $T_{a}[$ Fix $R \cap$ Fix $T]=$ Fix $R \cap$ Fix $T$. We find from the calculation

$$
\begin{aligned}
T_{a}\left(q, 0,0, q_{k+1}^{+}, 0, p, 0,0, p_{k+1}^{+}, 0\right) \\
=\left(q, \mathcal{R}_{a}(0,0), \mathcal{R}_{2 a}\left(q_{k+1}^{+}, 0\right), p, \mathcal{R}_{a}(0,0), \mathcal{R}_{2 a}\left(p_{k+1}^{+}, 0\right)\right) \\
=\left(q, 0,0, \cos ((k+1) a) q_{k+1}^{+}, \sin ((k+1) a) q_{k+1}^{+}\right. \\
\left.\quad p, 0,0, \cos ((k+1) a) p_{k+1}^{+}, \sin ((k+1) a) p_{k+1}^{+}\right)
\end{aligned}
$$

that this criterion is met if and only if $a=n \pi /(k+1)$ and that $T_{a}=U_{2}$ when $n$ is odd and $T_{a}$ is the identity when $n$ is even. A similar calculation yields the result for $U_{1}$ when $k$ is even.

We now focus upon the four-dimensional reduced system of equations in the invariant subspace Fix $R$; for notational simplicity we write $\left(q_{1}, q_{2}, p_{1}, p_{2}\right)$ for $\left(q_{k}^{+}, q_{k+1}^{+}, p_{k}^{+}, p_{k+1}^{+}\right)$and retain the notation $\Upsilon$ for the canonical 2 -form on $\mathbb{R}^{4}$. The reduced Hamiltonian system is reversible with reverser $S:\left(q_{1}, q_{2}, p_{1}, p_{2}\right) \mapsto\left(q_{1}, q_{2},-p_{1},-p_{2}\right)$ and has the additional $\mathbb{Z}_{2}$-symmetries specified in Proposition 3.1. We exploit these symmetries repeatedly in calculating the reduced system of equations (and of course in discussing their solutions). The next result, which gives a criterion for the appearance of a monomial in the Hamiltonian, is also important in this respect.

Proposition 3.2 The coefficient of the monomial $q_{1}^{n_{1}} p_{1}^{n_{2}} q_{2}^{n_{3}} p_{2}^{n_{4}}$ in the Taylor expansion of the Hamiltonian function vanishes unless $(i) n_{2}+n_{4}, n_{1}+n_{2}$ and $n_{3}+n_{4}$ are all even; or (ii) $n_{2}+n_{4}$ is even and $\left(n_{1}+n_{2}\right) /\left(n_{3}+n_{4}\right)=(k+1) / k$.

Proof It follows from the reversibility that the Hamiltonian function is invariant under the transformation $\left(p_{1}, p_{2}\right) \mapsto\left(-p_{1},-p_{2}\right)$, and this condition yields the criterion that $n_{2}+n_{4}$ should be even. Recalling that $\left(q_{1}, p_{2}\right)$ and $\left(q_{2}, p_{2}\right)$ are coordinates associated at linear level with the components in respectively the $k$ th and $(k+1)$ th Fourier modes, we obtain the remaining criteria by the combinatorics of the $k: k+1$ mode interaction. 
Corollary 3.3 Suppose that $k \geq 2$. The subspaces $\left\{\left(q_{1}, p_{1}\right)=(0,0)\right\}$ and $\left\{\left(q_{2}, p_{2}\right)=(0,0)\right\}$ are invariant under the flow generated by the Taylor expansion of the Hamiltonian vector field to every order; these subspaces are equipped with the $\mathbb{Z}_{2}$-symmetries $U_{2}:\left(q_{2}, p_{2}\right) \mapsto\left(-q_{2},-p_{2}\right)$ and $U_{1}:\left(q_{1}, p_{1}\right) \mapsto\left(-q_{1},-p_{1}\right)$ respectively.

Proof The invariance of the $\left(q_{1}, p_{1}\right)$ and $\left(q_{2}, p_{2}\right)$ coordinate planes follows from the fact that the Taylor expansion of the Hamiltonian contains no terms which are linear in $\left(q_{1}, p_{1}\right)$ or $\left(q_{2}, p_{2}\right)$. Moreover, it also contains no odd monomials in $\left(q_{1}, p_{1}\right)$ or $\left(q_{2}, p_{2}\right)$, so that $U_{1}$ and $U_{2}$ are symmetries in respectively the $\left(q_{1}, p_{1}\right)$ and $\left(q_{2}, p_{2}\right)$ coordinate planes.

Notice that we cannot conclude that the subspaces $\left\{\left(q_{1}, p_{1}\right)=(0,0)\right\}$ and $\left\{\left(q_{2}, p_{2}\right)=(0,0)\right\}$ are invariant under the flow generated by the Hamiltonian vector field itself in this manner since the Hamiltonian is not analytic; the invariance of $\left\{\left(q_{1}, p_{1}\right)=(0,0)\right\}$ for odd values of $k$ and $\left\{\left(q_{2}, p_{2}\right)=(0,0)\right\}$ for even values of $k$ is however an immediate consequence of Proposition 3.1.

We now have a convenient coordinate system with which to discuss the complete unfolding of the codimension-two point $P_{k, k+1}$ in $(\beta, \alpha)$-parameter space by means of a four-dimensional centre manifold. The centre-manifold reduction captures in particular the Hamiltonian $0^{2} 0^{2}$ resonance at the point $P_{k, k+1}$ itself, where the zero eigenspace and generalised eigenspace are respectively two and four dimensional, the Hamiltonian semisimple $1: 1$ resonance along the local part of curve $I_{k, k+1}$, where two geometrically double imaginary eigenvalues exist, and the Hamiltonian real semisimple 1: 1 resonance along the local part of the curve $R_{k, k+1}$, where two geometrically double real eigenvalues exist (see Figure 2). Our primary interest lies in homoclinic bifurcation associated with the Hamiltonian real semisimple $1: 1$ resonance on the local part of $R_{k, k+1}$, and we proceed by choosing values of $\left(\epsilon_{1}, \epsilon_{2}\right) \in \tilde{\Lambda}$ in a fashion which enables us to access these curves effectively.

The local part of $R_{k, k+1}$ is calculated by expanding the parameterisation (8), (9) of the curve $R_{i, j}$ near $s=0$. We find that the reduced system of equations at $P_{k, k+1}$ has a pair of semisimple real eigenvalues $\pm \mu$ when

$$
\begin{aligned}
& \beta=P_{k, k+1}^{\beta}+\hat{\beta}_{k, k+1} \mu^{2}+O\left(\mu^{4}\right), \\
& \alpha=P_{k, k+1}^{\alpha}+\hat{\alpha}_{k, k+1} \mu^{2}+O\left(\mu^{4}\right),
\end{aligned}
$$

where

$$
\begin{gathered}
\hat{\beta}_{k, k+1}=\frac{\operatorname{coth} k \nu}{2 k\left(k^{2}-(k+1)^{2}\right) \nu^{3}}-\frac{\operatorname{coth}(k+1) \nu}{2(k+1)\left(k^{2}-(k+1)^{2}\right) \nu^{3}} \\
+\frac{\operatorname{coth}^{2} k \nu}{2\left(k^{2}-(k+1)^{2}\right) \nu^{2}}-\frac{\operatorname{coth}^{2}(k+1) \nu}{2\left(k^{2}-(k+1)^{2}\right) \nu^{2}}, \\
\hat{\alpha}_{k, k+1}=-\frac{1}{2}+\frac{\left(2 k^{2}-(k+1)^{2}\right) \operatorname{coth} k \nu}{2 k\left(k^{2}-(k+1)^{2}\right) \nu}+\frac{\left(k^{2}-2(k+1)^{2}\right) \operatorname{coth}(k+1) \nu}{2(k+1)\left(k^{2}-(k+1)^{2}\right) \nu} \\
-\frac{(k+1)^{2} \operatorname{coth}^{2} k \nu}{2\left(k^{2}-(k+1)^{2}\right)}+\frac{k^{2} \operatorname{coth}^{2}(k+1) \nu}{2\left(k^{2}-(k+1)^{2}\right)} .
\end{gathered}
$$

To study codimension-one bifurcation phenomena associated with this curve we therefore write

$$
\epsilon_{1}=\hat{\beta}_{k, k+1} \mu^{2}, \quad \epsilon_{2}=\left(\hat{\alpha}_{k, k+1}+\delta\right) \mu^{2},
$$


where $\delta$ and $\mu$ are small positive numbers. The former parameter $\delta$ plays the role of a bifurcation parameter (varying $\delta$ through zero from above we cross the critical curve $R_{k, k+1}$ in parameter space near $P_{k, k+1}$ from above), while the latter parameter $\mu$ indicates the distance from the point $P_{k, k+1}$. Using the parameterisation (33) and Proposition 3.2, we find that the quadratic part of the reduced Hamiltonian function is given by

$$
\tilde{H}_{\mathrm{L}}^{\mu, \delta}(q, p)=\frac{1}{2}\left(1+\omega^{\mu, \delta}\right) p_{1}^{2}+\frac{1}{2}\left(1+\omega^{\mu, \delta}\right) p_{2}^{2}-\frac{\mu^{2}}{2}\left(c_{1}+\omega^{\mu, \delta}\right) q_{1}^{2}-\frac{\mu^{2}}{2}\left(c_{2}+\omega^{\mu, \delta}\right) q_{2}^{2},
$$

where the symbol $\omega^{\mu, \delta}$ denotes various, possibly different smooth functions of $\mu$ and $\delta$ which are $O\left(\mu^{2}\right)$. The method explained by Groves \& Mielke [9, Appendix B] yields the formulae

$$
c_{1}=-\left.\frac{2}{\mu^{2}} H_{2}^{\mu, \delta}\left(e_{1}, e_{1}\right)\right|_{\mu=0}=1+C_{1} \delta, \quad c_{2}=-\left.\frac{2}{\mu^{2}} H_{2}^{\mu, \delta}\left(e_{2}, e_{2}\right)\right|_{\mu=0}=1+C_{2} \delta
$$

in which the notation $H_{n}^{\epsilon}=\frac{1}{n !} \mathrm{d}^{n} H^{\epsilon}[0]$ has been used. The coefficients $C_{1}, C_{2}$ are readily computed and are stated in Appendix A; they satisfy

$$
C_{2}<C_{1}<0 \text {. }
$$

The next step is to compute the 'nonlinear' part of the reduced Hamiltonian and hence write down the reduced equations near the codimension-two points $P_{k, k+1}$; we treat the cases $k=1$ and $k \geq 2$ separately.

\subsection{The reduced equations near $P_{1,2}$}

To compute the leading-order terms in the 'nonlinear' part of the reduced Hamiltonian, we note that the reduced system is reversible and has the $\mathbb{Z}_{2}$-symmetry $T_{1}:\left(q_{1}, q_{2}, p_{1}, p_{2}\right) \mapsto$ $\left(-q_{1}, q_{2},-p_{1}, p_{2}\right)$ and the $\mathbb{Z}_{2}$-symmetry $U_{2}:\left(q_{2}, p_{2}\right) \mapsto\left(-q_{2},-p_{2}\right)$ in the invariant subspace Fix $T_{1}=\left\{\left(q_{1}, p_{1}\right)=(0,0)\right\}$. It follows that the only monomial in $q$ in the cubic part of its Hamiltonian is $q_{1}^{2} q_{2}$, so that

$$
\tilde{H}_{\mathrm{NL}}^{\mu, \delta}\left(q_{1}, q_{2}, p_{1}, p_{2}\right)=\frac{1}{2}\left(c+\omega^{\mu, \delta}\right) q_{1}^{2} q_{2}+O\left(|p||(q, p)|^{2}\right)+O\left(|(q, p)|^{4}\right),
$$

and that the cubic part of its Hamiltonian vanishes in the invariant subspace $\left\{\left(q_{1}, p_{1}\right)=(0,0)\right\}$, so that

$$
\tilde{H}_{\mathrm{NL}}^{\mu, \delta}\left(0, q_{2}, 0, p_{2}\right)=\frac{1}{4}\left(d+\omega^{\mu, \delta}\right) q_{2}^{4}+O\left(\left|p_{2}\right|^{2}\left|\left(q_{2}, p_{2}\right)\right|^{2}\right)+O\left(\left|\left(q_{2}, p_{2}\right)\right|^{6}\right) .
$$

According to the method explained by Groves \& Mielke [9, Appendix B], the coefficients $c$ and $d$ are given by the formulae

$$
\begin{aligned}
c & =6 H_{3}^{0}\left(e_{1}, e_{1}, e_{2}\right), \\
d & =4 H_{4}^{0}\left(e_{2}, e_{2}, e_{2}, e_{2}\right)+6 H_{3}^{0}\left(e_{2}, e_{2}, h_{0,0020}\right),
\end{aligned}
$$

where $h_{0,0020}$ is the coefficient of $q_{2}^{2}$ in the Taylor-series expansion of $h(q, p, 0)$. Expressions for $c$ and $d$ are presented in Appendix A; we find that $c<0, d>0$. 
The reduced equations are

$$
\begin{aligned}
& \dot{q}_{1}=\partial_{p_{1}} \tilde{H}^{\mu, \delta}=\left(1+\omega^{\mu, \delta}\right) p_{1}+\mathcal{R}_{1}, \\
& \dot{q}_{2}=\partial_{p_{2}} \tilde{H}^{\mu, \delta}=\left(1+\omega^{\mu, \delta}\right) p_{2}+\mathcal{R}_{2}, \\
& \dot{p}_{1}=-\partial_{q_{1}} \tilde{H}^{\mu, \delta}=\mu^{2}\left(1+C_{1} \delta+\omega^{\mu, \delta}\right) q_{1}-\left(c+\omega^{\mu, \delta}\right) q_{1} q_{2}+\mathcal{R}_{3}, \\
& \dot{p}_{2}=-\partial_{q_{2}} \tilde{H}^{\mu, \delta}=\mu^{2}\left(1+C_{2} \delta+\omega^{\mu, \delta}\right) q_{2}-\frac{1}{2}\left(c+\omega^{\mu, \delta}\right) q_{1}^{2}+\mathcal{R}_{4},
\end{aligned}
$$

in which the dot denotes differentiation with respect to $z$ and the remainder terms $\mathcal{R}_{i}$ satisfy the estimates

$$
\begin{aligned}
& \mathcal{R}_{1}, \mathcal{R}_{2}=O\left(|(q, p)|^{2}\right), \\
& \mathcal{R}_{3}, \mathcal{R}_{4}=O(|p||(q, p)|)+O\left(|(q, p)|^{3}\right)
\end{aligned}
$$

and have the symmetries

$$
\begin{aligned}
\left(\mathcal{R}_{1}, \mathcal{R}_{2}, \mathcal{R}_{3}, \mathcal{R}_{4}\right)(q,-p) & =\left(-\mathcal{R}_{1},-\mathcal{R}_{2}, \mathcal{R}_{3}, \mathcal{R}_{4}\right)(q, p) \\
\left(\mathcal{R}_{1}, \mathcal{R}_{2}, \mathcal{R}_{3}, \mathcal{R}_{4}\right)\left(-q_{1}, q_{2},-p_{1}, p_{2}\right) & =\left(-\mathcal{R}_{1}, \mathcal{R}_{2},-\mathcal{R}_{3}, \mathcal{R}_{4}\right)\left(q_{1}, q_{2}, p_{1}, p_{2}\right) \\
\left(\mathcal{R}_{2}, \mathcal{R}_{4}\right)\left(0,-q_{2}, 0,-p_{2}\right) & =\left(-\mathcal{R}_{2},-\mathcal{R}_{4}\right)\left(0, q_{2}, 0, p_{2}\right)
\end{aligned}
$$

Introducing the scaled variables

$$
Z=\mu z, \quad\left(Q_{1}(Z), Q_{2}(Z)\right)=\frac{1}{\mu^{2}}\left(q_{1}(z), q_{2}(z)\right), \quad\left(P_{1}(Z), P_{2}(Z)\right)=\frac{1}{\mu^{3}}\left(p_{1}(z), p_{2}(z)\right),
$$

we find from equations (35)-(38) that

$$
\begin{aligned}
Q_{1}^{\prime} & =P_{1}+O(\mu), \\
Q_{2}^{\prime} & =P_{2}+O(\mu), \\
P_{1}^{\prime} & =\left(1+C_{1} \delta\right) Q_{1}-c Q_{1} Q_{2}+O(\mu), \\
P_{2}^{\prime} & =\left(1+C_{2} \delta\right) Q_{2}-\frac{1}{2} c Q_{1}^{2}+O(\mu),
\end{aligned}
$$

in which the prime denotes differentiation with respect to $Z$. In the limit $\mu \rightarrow 0$ equations (39)-(42) admit the invariant subspace $\left\{\left(Q_{1}, P_{1}\right)=\sqrt{2}\left(Q_{2}, P_{2}\right)\right\}$, which contains the explicit homoclinic solution

$$
\left(Q_{2}(Z), P_{2}(Z)\right)=\left(\frac{3}{2 c} \operatorname{sech}^{2}\left(\frac{Z}{2}\right),-\frac{3}{2 c} \tanh \left(\frac{Z}{2}\right) \operatorname{sech}^{2}\left(\frac{Z}{2}\right)\right)
$$

at $\delta=0$, from which a second explicit homoclinic solution is obtained using the reflector $T_{1}$ (that is, reversing the sign of $\left(Q_{1}, P_{1}\right)$ ).

The next step is to show that the homoclinic orbit given by (43) is transverse in the usual sense in Hamiltonian-systems theory, namely that the tangent spaces to the stable and unstable manifolds of the zero equilibrium span the tangent space to the zero energy surface of the Hamiltonian function at each point of the homoclinic orbit. This property holds if and only if the only common tangent vector to the stable and unstable manifolds at each point of the homoclinic orbit is the 'time'-derivative of the homoclinic orbit itself. 
Proposition 3.4 The formula (43) defines a transverse homoclinic solution to the Hamiltonian system (39)-(42) at $\mu=0, \delta=0$.

Proof A straightforward linear change of variables transforms equations (39)-(42) with $\mu=0$, $\delta=0$ into the same equations with $c$ replaced by 1 . It therefore suffices to prove the proposition for $c=1$, and this result has been obtained by Yew [24, Lemma 2.2], who examined the equation of variations around the homoclinic orbit and showed that its only globally bounded solution is the 'time'-derivative of the homoclinic orbit itself.

Since transversality is an open phenomenon, we obtain the following result as an immediate consequence of Proposition 3.4.

Corollary 3.5 There exists a branch of transverse homoclinic solutions to equations (39)-(42), parameterised by values of $(\mu, \delta)$ in a neighbourhood of the origin in $\mathbb{R}^{2}$, which contains the explicit solution (43) at $\mu=0, \delta=0$. A second branch of transverse homoclinic solutions is obtained from the first using the reflector $T_{1}$.

Let us now examine the flow associated with the reduced Hamiltonian system in the invariant subspace $\left\{\left(q_{1}, p_{1}\right)=(0,0)\right\}$, in which the reduced equations are

$$
\begin{aligned}
& \dot{q}_{2}=\partial_{p_{2}} \tilde{H}^{\mu, \delta}=\left(1+\omega^{\mu, \delta)} p_{2}+\mathcal{R}_{2},\right. \\
& \dot{p}_{2}=-\partial_{q_{2}} \tilde{H}^{\mu, \delta}=\mu^{2}\left(1+C_{2} \delta+\omega^{\mu, \delta}\right) q_{2}-\left(d+\omega^{\mu, \delta}\right) q_{2}^{3}+\mathcal{R}_{4},
\end{aligned}
$$

where the remainder terms $\mathcal{R}_{i}$ satisfy the estimates

$$
\mathcal{R}_{1}=O\left(\left|\left(q_{2}, p_{2}\right)\right|^{3}\right), \quad \mathcal{R}_{2}=O\left(\left|p_{2}\right|\left|\left(q_{2}, p_{2}\right)\right|^{2}\right)+O\left(\left|\left(q_{2}, p_{2}\right)\right|^{5}\right)
$$

and have the symmetries

$$
\left(\mathcal{R}_{1}, \mathcal{R}_{2}\right)\left(q_{2},-p_{2}\right)=\left(-\mathcal{R}_{1}, \mathcal{R}_{2}\right)\left(q_{2}, p_{2}\right), \quad\left(\mathcal{R}_{1}, \mathcal{R}_{2}\right)\left(-q_{2},-p_{2}\right)=\left(-\mathcal{R}_{1},-\mathcal{R}_{2}\right)\left(q_{2}, p_{2}\right)
$$

Introducing the scaled variables

$$
Z=\mu z, \quad Q_{2}(Z)=\frac{1}{\mu} q_{2}(z), \quad P_{2}(Z)=\frac{1}{\mu^{2}} p_{2}(z),
$$

we find that

$$
\begin{aligned}
Q_{2}^{\prime} & =P_{2}+O(\mu), \\
P_{2}^{\prime} & =\left(1+C_{2} \delta\right) Q_{2}-d Q_{2}^{3}+O(\mu),
\end{aligned}
$$

in which the prime denotes differentiation with respect to $Z$. In the limit $\mu \rightarrow 0$ these equations admit the explicit homoclinic solution

$$
\begin{aligned}
& \left(Q_{2}(Z), P_{2}(Z)\right)= \\
& \quad\left(\left(\frac{2\left(1+C_{2} \delta\right)}{d}\right)^{1 / 2} \operatorname{sech}\left(1+C_{2} \delta\right)^{1 / 2} Z,-\left(\frac{2\left(1+C_{2} \delta\right)^{2}}{d}\right)^{1 / 2} \tanh \left(1+C_{2} \delta\right)^{1 / 2} Z \operatorname{sech}\left(1+C_{2} \delta\right)^{1 / 2} Z\right),
\end{aligned}
$$


from which a further explicit homoclinic solution is obtained using the reflector $U_{2}$ (that is, reversing the sign of the right-hand side of (46)). It is a straightforward exercise to show that (46) defines a transverse homoclinic solution to the two-dimensional Hamiltonian system under consideration here, and the following proposition is a direct consequence of this observation.

Proposition 3.6 The Hamiltonian system (44)-(45) admits a branch of homoclinic solutions, parameterised by values of $(\mu, \delta)$ is a neighbourhood of the origin in $\mathbb{R}^{2}$, which contains the explicit solution (46) at $\mu=0$; a further branch is obtained by applying the reflector $U_{2}$ to this basic branch.

\subsection{The reduced equations near $P_{k, k+1}$ for $k \geq 2$}

To compute the leading-order terms in the 'nonlinear' part of the reduced Hamiltonian, we note that according to Proposition 3.2 the cubic part of the Hamiltonian is identically zero and the only monomials in $q$ in its quartic part are $q_{1}^{4}, q_{2}^{4}$ and $q_{1}^{2} q_{2}^{2}$. The 'nonlinear' part of the Hamiltonian is therefore given by

$$
\begin{aligned}
& \tilde{H}_{\mathrm{NL}}^{\mu, \delta}\left(q_{1}, q_{2}, p_{1}, p_{2}\right) \\
& \quad=\frac{1}{4}\left(c_{1}+\omega^{\mu, \delta}\right) q_{1}^{4}+\frac{1}{4}\left(c_{2}+\omega^{\mu, \delta}\right) q_{2}^{4}+\frac{1}{2}\left(c_{3}+\omega^{\mu, \delta}\right) q_{1}^{2} q_{2}^{2}+O\left(|p|^{2}|(q, p)|^{2}\right)+O\left(|(q, p)|^{6}\right),
\end{aligned}
$$

in which the coefficients $c_{1}, c_{2}, c_{3}$ are given by the formulae

$$
\begin{aligned}
& c_{1}=4 H_{4}^{0}\left(e_{1}, e_{1}, e_{1}, e_{1}\right)+6 H_{3}^{0}\left(e_{1}, e_{1}, h_{0,2000}\right), \\
& c_{2}=4 H_{4}^{0}\left(e_{2}, e_{2}, e_{2}, e_{2}\right)+6 H_{3}^{0}\left(e_{2}, e_{2}, h_{0,0020}\right), \\
& c_{3}=12 H_{4}^{0}\left(e_{1}, e_{1}, e_{2}, e_{2}\right)+6 H_{3}^{0}\left(e_{1}, e_{2}, h_{0,1010}\right)+6 H_{3}^{0}\left(e_{2}, e_{2}, h_{0,2000}\right)
\end{aligned}
$$

and $h_{0,2000}, h_{0,0020}, h_{0,1010}$ are the coefficients of respectively $q_{1}^{2}, q_{2}^{2}, q_{1} q_{2}$ in the Taylor-series expansion of $h(q, p, 0)$. Formulae for $c_{1}, c_{2}, c_{3}$ are presented in Appendix A; we find that they are all positive. The reduced equations are

$$
\begin{aligned}
& \dot{q}_{1}=\partial_{p_{1}} \tilde{H}^{\mu, \delta}=\left(1+\omega^{\mu, \delta}\right) p_{1}+\mathcal{R}_{1}, \\
& \dot{q}_{2}=\partial_{p_{2}} \tilde{H}^{\mu, \delta}=\left(1+\omega^{\mu, \delta}\right) p_{2}+\mathcal{R}_{2}, \\
& \dot{p}_{1}=-\partial_{q_{1}} \tilde{H}^{\mu, \delta}=\mu^{2}\left(1+C_{1} \delta+\omega^{\mu, \delta}\right) q_{1}-\left(c_{1}+\omega^{\mu, \delta}\right) q_{1}^{3}-\left(c_{3}+\omega^{\mu, \delta}\right) q_{1} q_{2}^{2}+\mathcal{R}_{3}, \\
& \dot{p}_{2}=-\partial_{q_{2}} \tilde{H}^{\mu, \delta}=\mu^{2}\left(1+C_{2} \delta+\omega^{\mu, \delta}\right) q_{2}-\left(c_{2}+\omega^{\mu, \delta}\right) q_{2}^{3}-\left(c_{3}+\omega^{\mu, \delta}\right) q_{1}^{2} q_{2}+\mathcal{R}_{4},
\end{aligned}
$$

in which the dot denotes differentiation with respect to $z$ and the remainder terms $\mathcal{R}_{i}$ satisfy the estimates

$$
\begin{aligned}
& \mathcal{R}_{1}, \mathcal{R}_{2}=O\left(|(q, p)|^{3}\right) \\
& \mathcal{R}_{3}, \mathcal{R}_{4}=O\left(|p||(q, p)|^{2}\right)+O\left(|(q, p)|^{5}\right)
\end{aligned}
$$

The reduced Hamiltonian system is reversible, so that

$$
\left(\mathcal{R}_{1}, \mathcal{R}_{2}, \mathcal{R}_{3}, \mathcal{R}_{4}\right)(q,-p)=\left(-\mathcal{R}_{1},-\mathcal{R}_{2}, \mathcal{R}_{3}, \mathcal{R}_{4}\right)(q, p)
$$


and has certain additional $\mathbb{Z}_{2}$-symmetries. For odd values of $k$ it has a $\mathbb{Z}_{2}$-symmetry with reflector $T_{1}:\left(q_{1}, q_{2}, p_{1}, p_{2}\right) \mapsto\left(-q_{1}, q_{2},-p_{1}, p_{2}\right)$ together with a $\mathbb{Z}_{2}$-symmetry with reflector $U_{2}:\left(q_{2}, p_{2}\right) \mapsto\left(-q_{2},-p_{2}\right)$ in the invariant subspace Fix $T_{1}=\left\{\left(q_{1}, p_{1}\right)=(0,0)\right\}$, so that

$$
\begin{aligned}
\left(\mathcal{R}_{1}, \mathcal{R}_{2}, \mathcal{R}_{3}, \mathcal{R}_{4}\right)\left(-q_{1}, q_{2},-p_{1}, p_{2}\right) & =\left(-\mathcal{R}_{1}, \mathcal{R}_{2},-\mathcal{R}_{3}, \mathcal{R}_{4}\right)\left(q_{1}, q_{2}, p_{1}, p_{2}\right), \\
\left(\mathcal{R}_{2}, \mathcal{R}_{4}\right)\left(0,-q_{2}, 0,-p_{2}\right) & =\left(-\mathcal{R}_{2},-\mathcal{R}_{4}\right)\left(0, q_{2}, 0, p_{2}\right)
\end{aligned}
$$

and similarly for even values of $k$ it has a $\mathbb{Z}_{2}$-symmetry with reflector $T_{2}:\left(q_{1}, q_{2}, p_{1}, p_{2}\right) \mapsto$ $\left(q_{1},-q_{2}, p_{1},-p_{2}\right)$ together with a $\mathbb{Z}_{2}$-symmetry with reflector $U_{1}:\left(q_{1}, p_{1}\right) \mapsto\left(-q_{1},-p_{1}\right)$ in the invariant subspace Fix $T_{2}=\left\{\left(q_{2}, p_{2}\right)=(0,0)\right\}$, so that

$$
\begin{aligned}
\left(\mathcal{R}_{1}, \mathcal{R}_{2}, \mathcal{R}_{3}, \mathcal{R}_{4}\right)\left(q_{1},-q_{2}, p_{1},-p_{2}\right) & =\left(\mathcal{R}_{1},-\mathcal{R}_{2}, \mathcal{R}_{3},-\mathcal{R}_{4}\right)\left(q_{1}, q_{2}, p_{1}, p_{2}\right), \\
\left(\mathcal{R}_{2}, \mathcal{R}_{4}\right)\left(-q_{1}, 0,-p_{1}, 0\right) & =\left(-\mathcal{R}_{2},-\mathcal{R}_{4}\right)\left(q_{1}, 0, p_{1}, 0\right) .
\end{aligned}
$$

Furthermore, Corollary 3.3 asserts that the subspaces $\left\{\left(q_{1}, p_{1}\right)=(0,0)\right\}$ and $\left\{\left(q_{2}, p_{2}\right)=(0,0)\right\}$ are both invariant under the flow generated by the Taylor expansion of the Hamiltonian vector field to every order; these subspaces are equipped with the $\mathbb{Z}_{2}$-symmetries with reflectors $U_{2}$ and $U_{1}$ respectively. Recall, however, that the Hamiltonian function is not analytic, and this fact prevents us from concluding that $\left\{\left(q_{1}, p_{1}\right)=(0,0)\right\}$ and $\left\{\left(q_{2}, p_{2}\right)=(0,0)\right\}$ are invariant subspaces under the flow generated by the Hamiltonian vector field itself (see the discussion following Corollary 3.3).

Introducing the scaled variables

$$
Z=\mu z, \quad\left(Q_{1}(Z), Q_{2}(Z)\right)=\frac{1}{\mu}\left(q_{1}(z), q_{2}(z)\right), \quad\left(P_{1}(Z), P_{2}(Z)\right)=\frac{1}{\mu^{2}}\left(p_{1}(z), p_{2}(z)\right),
$$

we find from equations (47)-(50) that

$$
\begin{aligned}
Q_{1}^{\prime} & =P_{1}+O(\mu), \\
Q_{2}^{\prime} & =P_{2}+O(\mu), \\
P_{1}^{\prime} & =\left(1+C_{1} \delta\right) Q_{1}-c_{1} Q_{1}^{3}-c_{3} Q_{1} Q_{2}^{2}+O(\mu), \\
P_{2}^{\prime} & =\left(1+C_{2} \delta\right) Q_{2}-c_{2} Q_{2}^{3}-c_{3} Q_{1}^{2} Q_{2}+O(\mu),
\end{aligned}
$$

in which the prime denotes differentiation with respect to $Z$. In the limit $\mu \rightarrow 0$ equations (51)-(54) admit the invariant subspace

$$
\left(Q_{1}, P_{1}\right)=\alpha\left(Q_{2}, P_{2}\right), \quad \alpha=\sqrt{\frac{c_{2}-c_{3}}{c_{1}-c_{3}}}
$$

provided that $\left(c_{2}-c_{3}\right) /\left(c_{1}-c_{3}\right)>0$, and this subspace contains the explicit homoclinic solution

$$
\left(Q_{2}(Z), P_{2}(Z)\right)=\left(\left(\frac{2\left(c_{1}-c_{3}\right)}{c_{1} c_{2}-c_{3}^{2}}\right)^{1 / 2} \operatorname{sech} Z,-\left(\frac{2\left(c_{1}-c_{3}\right)}{c_{1} c_{2}-c_{3}^{2}}\right)^{1 / 2} \operatorname{sech} Z \tanh Z\right)
$$

at $\delta=0$. (The quantity within the square root is equal to twice the reciprocal of $c_{2}+c_{3} \alpha^{2}$ and is therefore positive.) A further explicit homoclinic solution is obtained using the reflector $T_{1}$ (that is, reversing the sign of $\left.\left(Q_{1}, P_{1}\right)\right)$ for odd values of $k$ or the reflector $T_{2}$ (that is, reversing the sign of $\left.\left(Q_{2}, P_{2}\right)\right)$ for even values of $k$. 
Proposition 3.7 The formula (55) defines a transverse homoclinic solution to the Hamiltonian system (51)-(54) at $\mu=0, \delta=0$ provided that

$$
\lambda=\frac{2\left(3 c_{1} c_{2}-2 c_{1} c_{3}-2 c_{2} c_{3}+c_{3}^{2}\right)}{c_{1} c_{2}-c_{3}^{2}}
$$

is not a positive integer.

Proof Linearising equations (51)-(54) around the homoclinic solution given by (55), we obtain the equations

$$
\left(\begin{array}{c}
-Q_{1}^{\prime \prime}+Q_{1} \\
-Q_{2}^{\prime \prime}+Q_{2}
\end{array}\right)-\frac{2\left(c_{1}-c_{3}\right)}{c_{1} c_{2}-c_{3}^{2}} \operatorname{sech}^{2}(Z)\left(\begin{array}{cc}
3 c_{1} \alpha^{2}+c_{3} & 2 \alpha c_{3} \\
2 \alpha c_{3} & 3 c_{2}+\alpha^{2} c_{3}
\end{array}\right)\left(\begin{array}{l}
Q_{1} \\
Q_{2}
\end{array}\right)=\left(\begin{array}{l}
0 \\
0
\end{array}\right) .
$$

The square matrix on the left-hand side of this equation is real and symmetric and therefore diagonalisable; it has two real eigenvalues

$$
\lambda_{1}=\frac{3\left(c_{1} c_{2}-c_{3}^{2}\right)}{c_{1}-c_{3}}, \quad \lambda_{2}=\frac{3 c_{1} c_{2}-2 c_{1} c_{3}-2 c_{2} c_{3}+c_{3}^{2}}{c_{1}-c_{3}} .
$$

It follows that there is a linear change of coordinates $\left(Q_{1}, Q_{2}\right) \mapsto\left(\tilde{Q}_{1}, \tilde{Q}_{2}\right)$ which transforms (56) into the decoupled system

$$
\tilde{Q}_{1}^{\prime \prime}=\tilde{Q}_{1}-6 \operatorname{sech}^{2}(Z) \tilde{Q}_{1}, \quad \tilde{Q}_{2}^{\prime \prime}=\tilde{Q}_{2}-\lambda \operatorname{sech}^{2}(Z) \tilde{Q}_{2} .
$$

The solution set of the first of these equations is spanned by the two linearly independent solutions

$$
\tilde{Q}_{1}(Z)=\operatorname{sech} Z \tanh Z, \quad \tilde{Q}_{1}(Z)=\frac{1}{4} \operatorname{sech} Z \tanh Z(6 Z-4 \operatorname{coth} Z+\sinh 2 Z),
$$

the latter of which is unbounded as $Z \rightarrow \pm \infty$. A result given by Yew [24, Lemma 2.3] shows that the second equation has no globally bounded solution for $\lambda<0$, and for $\lambda=0$ its solution set is clearly spanned by the two linearly independent, unbounded solutions $Q_{1}(Z)=\mathrm{e}^{Z}$ and $Q_{1}(Z)=\mathrm{e}^{-Z}$. Turning now to the case $\lambda>0$, notice that the change of independent variable $T=\tanh Z$ transforms the second equation into the Legendre equation

$$
\left(1-T^{2}\right) \frac{\mathrm{d}^{2} \tilde{Q}_{2}}{\mathrm{~d} T^{2}}-2 T \frac{\mathrm{d} \tilde{Q}_{2}}{\mathrm{~d} T}-\left(r(r+1)-\frac{1}{1-T^{2}}\right) \tilde{Q}_{2}=0, \quad r=\frac{1}{2}(\sqrt{1+\lambda}-1) .
$$

This equation has two linearly independent solutions, namely the associated Legendre functions of the first and second kind $P_{r}^{1}(T), Q_{r}^{1}(T)$, which are unbounded at respectively $T=-1$ and $T=1$ provided that $r$ is not an integer. Returning to the original independent variable $Z$, we find that the corresponding solutions are unbounded respectively as $Z \rightarrow-\infty$ and $Z \rightarrow \infty$ provided that $r$ is not an integer, and it follows from the assumption that $\lambda \notin \mathbb{Z}$ that this condition is met.

We conclude that the equation of variations about the homoclinic solution given by formula (55) has only one linearly independent solution which is bounded for $Z \in \mathbb{R}$, namely the $Z$ derivative of the homoclinic solution, which is therefore transverse.

Using the expressions for $c_{1}, c_{2}$ and $c_{3}$ given in Appendix A one can verify that $\left(c_{2}-c_{3}\right) /\left(c_{1}-\right.$ $\left.c_{3}\right)>0$ and that $\lambda$ is not a positive integer. 
Corollary 3.8 There exists a branch of transverse homoclinic solutions to equations (51)-(54), parameterised by values of $(\mu, \delta)$ in a neighbourhood of the origin in $\mathbb{R}^{2}$, which contains the explicit solution (55) at $\mu=0, \delta=0$. A further branch of transverse homoclinic solutions is obtained from the first using the reflector $T_{1}$ for odd values of $k$ or $T_{2}$ for even values of $k$.

In the limit $\mu \rightarrow 0$ equations (51)-(54) admit the invariant subspace $\left\{\left(Q_{2}, P_{2}\right)=(0,0)\right\}$, which contains the explicit homoclinic solutions

$$
\begin{aligned}
& \left(Q_{1}(Z), P_{1}(Z)\right)= \\
& \quad \pm\left(\left(\frac{2\left(1+C_{1} \delta\right)}{c_{1}}\right)^{1 / 2} \operatorname{sech}\left(1+C_{1} \delta\right)^{1 / 2} Z,-\left(\frac{2\left(1+C_{1} \delta\right)^{2}}{c_{1}}\right)^{1 / 2} \tanh \left(1+C_{1} \delta\right)^{1 / 2} Z \operatorname{sech}\left(1+C_{1} \delta\right)^{1 / 2} Z\right)
\end{aligned}
$$

and the invariant subspace $\left\{\left(Q_{1}, P_{1}\right)=(0,0)\right\}$, which contains the explicit homoclinic solutions

$$
\begin{aligned}
& \left(Q_{2}(Z), P_{2}(Z)\right)= \\
& \quad \pm\left(\left(\frac{2\left(1+C_{2} \delta\right)}{c_{2}}\right)^{1 / 2} \operatorname{sech}\left(1+C_{2} \delta\right)^{1 / 2} Z,-\left(\frac{2\left(1+C_{2} \delta\right)^{2}}{c_{2}}\right)^{1 / 2} \tanh \left(1+C_{2} \delta\right)^{1 / 2} Z \operatorname{sech}\left(1+C_{2} \delta\right)^{1 / 2} Z\right) .
\end{aligned}
$$

Proposition 3.9 The formulae (58), (59) define transverse homoclinic solutions to the Hamiltonian system (51)-(54) at $\mu=0$ provided that $8 c_{3} / c_{1}$ is not an integer.

Proof It suffices to establish this result for $\delta=0$. Linearising equations (51)-(54) around one of the homoclinic solution given by formula (58), we obtain the equations

$$
\ddot{Q}_{1}=Q_{1}-6 \operatorname{sech}^{2}(Z) Q_{1}, \quad \ddot{Q}_{2}=Q_{2}-\frac{8 c_{3}}{c_{1}} \operatorname{sech}^{2}(Z) Q_{2} .
$$

Comparing this formula with (57) and using the method explained in the proof of Proposition 3.7, we find that the equation of variations about one of the homoclinic solutions given by formula (58) has only one linearly independent solution which is bounded for $Z \in \mathbb{R}$, namely the $Z$ derivative of the homoclinic solution, which is therefore transverse. The same argument shows that the homoclinic solutions given by formula (59) are also transverse.

Using the formulae for $c_{1}$ and $c_{3}$ given in Appendix A, one can verify that $8 c_{3} / c_{1}$ is not a positive integer.

Proposition 3.10 The Hamiltonian system (51)-(54) admits

(1) two branches of homoclinic solutions, parameterised by values of $(\mu, \delta)$ in a neighbourhood of the origin in $\mathbb{R}^{2}$, which contain the explicit solutions (58) at $\mu=0$;

(2) two branches of homoclinic solutions, parameterised by values of $(\mu, \delta)$ in a neighbourhood of the origin in $\mathbb{R}^{2}$, which contain the explicit solutions (59) at $\mu=0$.

Notice that Proposition 3.10(1) is an immediate consequence of the invariance of the subspace Fix $T_{2}=\left\{\left(q_{2}, p_{2}\right)=(0,0)\right\}$ for even values of $k$ : it is a straightforward exercise to show that formula (58) defines a transverse homoclinic solution to the two-dimensional Hamiltonian system in this subspace. Similarly, Proposition 3.10(2) follows from the invariance of the subspace Fix $T_{2}=\left\{\left(q_{1}, p_{1}\right)=(0,0)\right\}$ for odd values of $k$. 


\section{Existence theory for multipulse homoclinic orbits}

\subsection{Homoclinic Lyapunov-Schmidt theory}

We begin by outlining the homoclinic Lyapunov-Schmidt theory developed by Lin [18] and Sandstede [21, §3] which is applied in Section 4.2 below to find multipulse homoclinic solutions to the reduced equations near the points $P_{k, k+1}$. Consider a Hamiltonian system

$$
\dot{u}=f^{\delta}(u)
$$

with Hamiltonian function $H^{\delta}(u)$, where $u$ and $\delta$ lie in a neighbourhood of the origin in respectively $\mathbb{R}^{4}$ and $\mathbb{R}$. We suppose that equation (60) has certain discrete symmetries, namely reversibility (with reverser $S$ ) and $\ell_{1}$ distinct $\mathbb{Z}_{2}$-symmetries (with reflectors $T_{1}, \ldots, T_{\ell_{1}}$ ). It is also supposed to have $\ell_{2}$ distinct transverse homoclinic orbits $u_{1}^{0}(t), \ldots, u_{\ell_{2}}^{0}(t)$ at $\delta=0$ which are not related to each other by means of the reflectors $T_{1}, \ldots, T_{\ell_{1}}$. Since transversality is an open phenomenon, these transverse homoclinic orbits persist for small, nonzero values of $\delta$, where they are denoted by $u_{1}^{\delta}(t), \ldots, u_{\ell_{2}}^{\delta}(t)$ and satisfy

$$
T_{u_{j}^{\delta}(t)} W^{\mathrm{u}} \cap T_{u_{j}^{\delta}(t)} W^{\mathrm{s}}=\operatorname{span}\left\{\dot{u}_{j}^{\delta}(t)\right\}, \quad t \in \mathbb{R} ;
$$

a consequence of the transversality is that the subspace

$$
\left[T_{u_{j}^{\delta}(t)} W^{\mathrm{u}}+T_{u_{j}^{\delta}(t)} W^{\mathrm{s}}\right]^{\perp}
$$

of $\mathbb{R}^{4}$ is one-dimensional for each $t \in \mathbb{R}$, being the linear span of the vector $\Psi_{j}^{\delta}(t)=\nabla H^{\delta}\left(u_{j}^{\delta}(t)\right)$. The Hamiltonian system (60) is thus equipped with a catalogue $u_{1}^{\delta}(t), \ldots, u_{\ell_{2}}^{\delta}(t)$ of basic 'primary' homoclinic orbits and an accompanying catalogue of reflectors $T_{1}, \ldots, T_{\ell_{1}}$; further transverse homoclinic orbits are obtained from the basic primary orbits using the reflectors.

The homoclinic bifurcation theory introduced below relies crucially upon information from the 'tails' of the primary homoclinic solutions. It is therefore necessary to obtain suitable estimates on their asymptotic behaviour as $t \rightarrow \infty$. We record here a result for four-dimensional systems which is suitable for this purpose; its proof has been given by Yew [24, Lemma 2.1 and Corollary 2.1].

Lemma 4.1 Suppose the four-dimensional dynamical system

$$
\dot{u}=f^{\delta}(u)
$$

has four real, nonzero eigenvalues $\pm \lambda_{1}^{\delta}, \pm \lambda_{2}^{\delta}$ whose corresponding eigenvectors $e_{1}^{\delta+}, e_{1}^{\delta-}, e_{2}^{\delta+}$, $e_{2}^{\delta-}$ are linearly independent and define $\lambda_{\mathrm{s}}=\min \left\{\left|\lambda_{1}^{\delta}\right|,\left|\lambda_{2}^{\delta}\right|\right\}$. Any solution $u^{\delta}(t)$ to this dynamical system which lies upon its two-dimensional local stable manifold satisfies the asymptotic estimate

$$
\begin{aligned}
& u^{\delta}(t)=b^{\delta} e_{1}^{\delta-} \mathrm{e}^{-\lambda_{1}^{\delta} t}+c^{\delta} e_{2}^{\delta-} \mathrm{e}^{-\lambda_{2}^{\delta} t}+O\left(\mathrm{e}^{-2 \lambda_{\mathrm{s}} t}\right), \\
& \dot{u}^{\delta}(t)=-b^{\delta} \lambda_{1}^{\delta} e_{1}^{\delta-} \mathrm{e}^{-\lambda_{1}^{\delta} t}-c^{\delta} \lambda_{2}^{\delta} e_{2}^{\delta-} \mathrm{e}^{-\lambda_{2}^{\delta} t}+O\left(\mathrm{e}^{-2 \lambda_{\mathrm{s}} t}\right)
\end{aligned}
$$

as $t \rightarrow \infty$, where the coefficients $b^{\delta}, d^{\delta}$ depend smoothly upon $\delta$. 


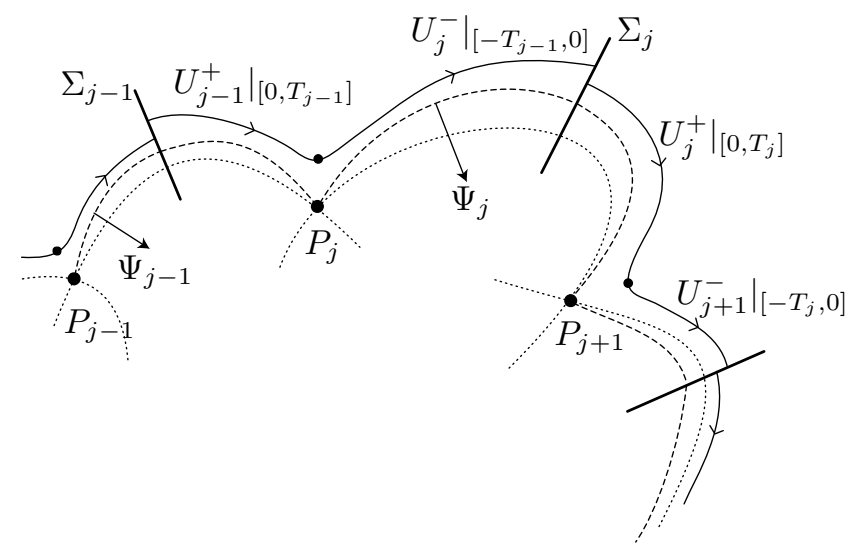

Figure 6: Piecewise continuous orbits $U_{j}^{ \pm}$for $\delta$ near 0 . The primary homoclinic orbits $u_{j}^{0}(t)$, $u_{j}^{\delta}(t)$ are depicted by respectively dashed and dotted curves.

Let us now consider a cycle of (not necessarily distinct) transverse homoclinic orbits $u_{1}^{\delta}(t)$, $\ldots, u_{N}^{\delta}(t)$ which connect $N+1$ copies of the zero equilibrium, denoted by $P_{1}, \ldots, P_{N+1}$. The following lemma was proved by Sandstede [21, §3]; its conclusions are illustrated in Figure 6.

Lemma 4.2 Let $\Sigma_{1}, \ldots \Sigma_{N}$ be codimension-one transverse sections to the orbits $u_{1}^{0}(t), \ldots u_{N}^{0}(t)$ at $t=0$, so that $\Psi_{j}^{\delta}(0)$ lies in $\Sigma_{j}$. For sufficiently small values of $\delta$ and any sequence $\boldsymbol{T}=$ $\left\{T_{j}\right\}_{j=1}^{N}$ with $T:=\min _{j=1, \ldots, N} T_{j}$ sufficiently large there exists a unique set $\left\{U_{j}^{ \pm}\right\}_{j=1}^{N}$ offunctions with the following properties:

(1) $U_{j}^{ \pm}$are solutions to (60) on respectively $\left[0, T_{j}\right]$ and $\left[-T_{j-1}, 0\right]$ which lie close to $u_{j}^{\delta}$;

(2) $U_{j}^{+}\left(T_{j}\right)=U_{j+1}^{-}\left(-T_{j}\right)$;

(3) $U_{j}^{-}(0)-U_{j}^{+}(0)=\xi_{j}(\boldsymbol{T}, \delta) \Psi_{j}^{\delta}(0)$.

The jump sizes $\xi_{j}(\boldsymbol{T}, \delta)$ are given by the formula

$$
\xi_{j}(\boldsymbol{T}, \delta)=\left\langle\Psi_{j}^{\delta}\left(-T_{j-1}\right), u_{j-1}^{\delta}\left(T_{j-1}\right)\right\rangle-\left\langle\Psi_{j}^{\delta}\left(T_{j}\right), u_{j+1}^{\delta}\left(-T_{j}\right)\right\rangle+\mathcal{R}_{j}(\boldsymbol{T}, \delta),
$$

where the remainder term $\mathcal{R}_{j}(\boldsymbol{T}, \delta)$ is a continuously differentiable function of $\boldsymbol{T}$ which depends smoothly upon $\delta$ and satisfies $\mathcal{R}_{j}(\boldsymbol{T}, \delta), \partial_{1} \mathcal{R}_{j}(\boldsymbol{T}, \delta)=O\left(\mathrm{e}^{-3 \lambda_{\mathrm{s}} T}\right)$ as $T \rightarrow \infty$.

The above lemma indicates the strategy we follow to find a multipulse homoclinic orbit which resembles a concatenation of the 'primary' homoclinic orbits $u_{1}^{\delta}(t), \ldots, u_{N}^{\delta}(t)$ : we attempt to solve the bifurcation equations

$$
\xi_{j}(\boldsymbol{T}, \delta)=0, \quad j=1, \ldots, N .
$$

Notice that $\delta=0, T_{j}=\infty, j=1, \ldots, N$ is a solution of these bifurcation equations which corresponds to $N$ unipulse homoclinic orbits, namely the primary orbits $u_{1}^{\delta}(t), \ldots, u_{N}^{\delta}(t)$. We therefore seek to apply the implicit function theorem at $T_{j}=\infty, j=1, \ldots, N$, introducing an appropriate change of variables to deal with the 'point' $\infty$. In this respect we note that $T_{1}, T_{N}=$ $\infty$ since we are seeking a homoclinic orbit, and the following proposition shows that it suffices to solve the first $N-1$ bifurcation equations; it is proved using the fact that the Hamiltonian function is a conserved quantity (see Sandstede, Jones \& Alexander [22, Lemma 3.2]). 
Proposition 4.3 The equations $\xi_{j}(\boldsymbol{T}, \delta)=0, j=1, \ldots, N-1$ imply that $\xi_{N}(\boldsymbol{T}, \delta)=0$.

The formulae for the jump sizes $\xi_{j}(\boldsymbol{T}, \delta)$ can be simplified by exploiting the structure of equation (60). The reversibility implies that

$$
u_{j}^{\delta}\left(-T_{j}\right)=S u_{j}^{\delta}\left(T_{j}\right)
$$

while the Hamiltonian structure shows that

$$
\begin{aligned}
\Psi_{j}^{\delta}\left(T_{j}\right) & =\nabla H^{\delta}\left(u_{j}^{\delta}(t)\right) \\
& =\nabla^{2} H^{\delta}(0) u_{j}^{\delta}\left(T_{j}\right)+O\left(\left|u_{j}^{\delta}\left(T_{j}\right)\right|^{2}\right)
\end{aligned}
$$

and similarly

$$
\begin{aligned}
\Psi_{j}^{\delta}\left(-T_{j-1}\right) & =\nabla^{2} H^{\delta}(0) u_{j}^{\delta}\left(-T_{j-1}\right)+O\left(\left|u_{j}^{\delta}\left(-T_{j-1}\right)\right|^{2}\right) \\
& =\nabla^{2} H^{\delta}(0) S u_{j}^{\delta}\left(T_{j-1}\right)+O\left(\left|u_{j}^{\delta}\left(T_{j-1}\right)\right|^{2}\right) .
\end{aligned}
$$

Furthermore, recall that the 'primary' homoclinic orbits $u_{1}^{\delta}(t), \ldots, u_{N}^{\delta}(t)$ are obtained by applying the reflectors $T_{1}, \ldots, T_{\ell_{1}}$ to the catalogue $u_{1}^{\delta}(t), \ldots, u_{\ell_{2}}^{\delta}(t)$ of basic primary homoclinic orbits: we can write

$$
u_{j}^{\delta}(t)=\gamma_{j}^{1} \ldots \gamma_{j}^{\ell_{1}} v_{j}^{\delta}(t), \quad \gamma_{j}^{i} \in\left\{I, T_{i}\right\}, v_{j}^{\delta} \in\left\{u_{1}^{\delta}, \ldots, u_{\ell_{2}}^{\delta}\right\} .
$$

Combining these observations and the fact that $\nabla^{2} H^{\delta}(0), S, T_{1}, \ldots, T_{\ell_{1}}$ are all self-adjoint and mutually commuting, we find that

$$
\xi_{j}(\boldsymbol{T}, \delta)=\left\langle M_{j-1}^{\delta} v_{j}^{\delta}\left(T_{j-1}\right), v_{j-1}^{\delta}\left(T_{j-1}\right)\right\rangle-\left\langle M_{j}^{\delta} v_{j}^{\delta}\left(T_{j}\right), v_{j+1}^{\delta}\left(T_{j}\right)\right\rangle+\tilde{\mathcal{R}}_{j}(\boldsymbol{T}, \delta),
$$

where

$$
M_{j}^{\delta}=\nabla^{2} H^{\delta}(0) S \gamma_{j}^{1} \ldots \gamma_{j}^{\ell_{1}} \gamma_{j+1}^{1} \ldots \gamma_{j+1}^{\ell_{1}}
$$

and the remainder term $\tilde{\mathcal{R}}_{j}(\boldsymbol{T}, \delta)$ satisfies the same asymptotic estimates as $\mathcal{R}_{j}(\boldsymbol{T}, \delta)$. Finally, we introduce the new variables $\eta_{j}=\left(\xi_{1}+\ldots+\xi_{j}\right) / 2, j=1, \ldots, N-1$, in terms of which the bifurcation equations are

$$
\eta_{j}(\boldsymbol{T}, \delta):=\left\langle M_{j}^{\delta} v_{j}^{\delta}\left(T_{j}\right), v_{j+1}^{\delta}\left(T_{j}\right)\right\rangle+\hat{\mathcal{R}}_{j}(\boldsymbol{T}, \delta)=0, \quad j=1, \ldots, N-1
$$

and the remainder term $\hat{\mathcal{R}}_{j}(\boldsymbol{T}, \delta)$ again satisfies the same asymptotic estimates as $\mathcal{R}_{j}(\boldsymbol{T}, \delta)$.

We now proceed to write down and study these bifurcation equations as they relate to the reduced Hamiltonian system near the points $P_{k, k+1}$. The equations and homoclinic orbits discussed here depend upon two parameters $\delta$ and $\mu$; the former plays the role of the bifurcation parameter in the homoclinic Lyapunov-Schmidt theory while the latter is treated as a small, fixed positive number. 


\subsection{Multipulse homoclinic orbits near $P_{k, k+1}$}

Our starting point in this section is the reduced system near the codimension-two point $P_{k, k+1}$, which is conveniently described in scaled coordinates by equations (39)-(42) for $k=1$ or equations (51)-(54) for $k \geq 2$. We consider the catalogue of basic primary orbits consisting of the single orbit $u_{k, k+1}^{\mu, \delta}(t)$ which, at $(\mu, \delta)=(0,0)$, has the explicit representation (43) for $k=1$ or (55) for $k \geq 2$, where we replace $Z$ by $t$ in keeping with the notation in Section 4.1 above. Its catalogue of reflectors also has just one entry, namely $T_{1}=\operatorname{diag}(-1,1,-1,1)$ for even values of $k$ or $T_{2}=\operatorname{diag}(1,-1,1,-1)$ for odd values of $k$.

The linear part of the reduced Hamiltonian system near $P_{k, k+1}$ is

$$
\begin{aligned}
Q_{1}^{\prime} & =\left(1+\omega^{\mu, \delta}\right) P_{1} \\
Q_{2}^{\prime} & =\left(1+\omega^{\mu, \delta}\right) P_{2} \\
P_{1}^{\prime} & =\left(1+C_{1} \delta+\omega^{\mu, \delta}\right) Q_{1} \\
P_{2}^{\prime} & =\left(1+C_{2} \delta+\omega^{\mu, \delta}\right) Q_{2}
\end{aligned}
$$

and the quadratic part of the reduced Hamiltonian function is

$$
\tilde{H}_{\mathrm{L}}^{\mu, \delta}(Q, P)=\frac{1}{2}\left(1+\omega^{\mu, \delta}\right) P_{1}^{2}+\frac{1}{2}\left(1+\omega^{\mu, \delta}\right) P_{2}^{2}-\frac{1}{2}\left(c_{1}+\omega^{\mu, \delta}\right) Q_{1}^{2}-\frac{1}{2}\left(c_{2}+\omega^{\mu, \delta}\right) Q_{2}^{2},
$$

where we recall that the symbol $\omega^{\mu, \delta}$ denotes various, possibly different smooth functions of $\mu$ and $\delta$ which are $O\left(\mu^{2}\right)$. This system has the eigenvalues $\pm\left(1+C_{1} \delta+\omega^{\mu, \delta}\right)^{1 / 2}, \pm\left(1+C_{2} \delta+\omega^{\mu, \delta}\right)^{1 / 2}$ with corresponding eigenvectors

$$
\left(\begin{array}{c}
\left(1+\omega^{\mu, \delta}\right)^{1 / 2} \\
0 \\
\pm\left(1+C_{1} \delta+\omega^{\mu, \delta}\right)^{1 / 2} \\
0
\end{array}\right), \quad\left(\begin{array}{c}
0 \\
\left(1+\omega^{\mu, \delta}\right)^{1 / 2} \\
0 \\
\pm\left(1+C_{2} \delta+\omega^{\mu, \delta}\right)^{1 / 2}
\end{array}\right)
$$

and using Lemma 4.1, we find that our basic primary homoclinic orbit has the asymptotic expansion

$$
\begin{aligned}
u_{k, k+1}^{\mu, \delta}(t)=b^{\mu, \delta} & \left(\begin{array}{c}
\left(1+\omega^{\mu, \delta}\right)^{1 / 2} \\
0 \\
-\left(1+C_{1} \delta+\omega^{\mu, \delta}\right)^{1 / 2} \\
0
\end{array}\right) \mathrm{e}^{-\left(1+C_{1} \delta+\omega^{\mu, \delta}\right)^{1 / 2} t} \\
& +d^{\mu, \delta}\left(\begin{array}{c}
0 \\
\left(1+\omega^{\mu, \delta}\right)^{1 / 2} \\
0 \\
-\left(1+C_{2} \delta+\omega^{\mu, \delta}\right)^{1 / 2}
\end{array}\right) \mathrm{e}^{-\left(1+C_{2} \delta+\omega^{\mu, \delta}\right)^{1 / 2} t}+O\left(\mathrm{e}^{-2 \lambda_{\mathrm{s}} t}\right)
\end{aligned}
$$

as $t \rightarrow \infty$, where $b^{\mu, \delta}, d^{\mu, \delta}$ are smooth functions of $\mu$ and $\delta$ which, according to the explicit formulae for $u_{k, k+1}^{0,0}(t)$, satisfy

$$
b^{0,0}=\left\{\begin{array}{ll}
\frac{6 \sqrt{2}}{c}, & k=1, \\
2\left(\frac{2\left(c_{1}-c_{3}\right)}{c_{1} c_{2}-c_{3}^{2}}\right)^{1 / 2}, & k \geq 2,
\end{array} \quad d^{0,0}= \begin{cases}\frac{6}{c}, & k=1 \\
2\left(\frac{2\left(c_{2}-c_{3}\right)}{c_{1} c_{2}-c_{3}^{2}}\right)^{1 / 2}, & k \geq 2\end{cases}\right.
$$


The above calculations show that

$M_{j}^{\mu, \delta}=\nabla^{2} \tilde{H}^{\mu, \delta}(0) R \gamma_{j} \gamma_{j+1}=-\operatorname{diag}\left(\delta_{j}\left(1+C_{1} \delta+\omega^{\mu, \delta}\right), 1+C_{2} \delta+\omega^{\mu, \delta}, \delta_{j}\left(1+\omega^{\mu, \delta}\right), 1+\omega^{\mu, \delta}\right)$

for odd values of $k$ and

$M_{j}^{\mu, \delta}=\nabla^{2} \tilde{H}^{\mu, \delta}(0) R \gamma_{j} \gamma_{j+1}=-\operatorname{diag}\left(1+C_{1} \delta+\omega^{\mu, \delta}, \delta_{j}\left(1+C_{2} \delta+\omega^{\mu, \delta}\right), 1+\omega^{\mu, \delta}, \delta_{j}\left(1+\omega^{\mu, \delta}\right)\right)$

for even values of $k$, where

$$
\delta_{j}= \begin{cases}1, & \gamma_{j}=\gamma_{j+1} \\ -1, & \gamma_{j} \neq \gamma_{j+1}\end{cases}
$$

It follows that

$$
\begin{aligned}
\left\langle M_{j}^{\mu, \delta} u_{k, k+1}^{\mu, \delta}\left(T_{j}\right), u_{k, k+1}^{\mu, \delta}\left(T_{j}\right)\right\rangle= & -2 \delta_{j}\left(1+C_{1} \delta+\omega_{1}^{\mu, \delta}\right)\left(b^{\mu, \delta}\right)^{2} \mathrm{e}^{-2\left(1+C_{1} \delta+\omega_{2}^{\mu, \delta}\right)^{1 / 2} T_{j}} \\
& -2\left(1+C_{2} \delta+\omega_{3}^{\mu, \delta}\right)\left(d^{\mu, \delta}\right)^{2} \mathrm{e}^{-\left(1+C_{2} \delta+\omega_{4}^{\mu, \delta}\right)^{1 / 2} T_{j}}+O\left(\mathrm{e}^{-3 \lambda_{\mathrm{s}} t}\right)
\end{aligned}
$$

for odd values of $k$ and

$$
\begin{aligned}
\left\langle M_{j}^{\mu, \delta} u_{k, k+1}^{\mu, \delta}\left(T_{j}\right), u_{k, k+1}^{\mu, \delta}\left(T_{j}\right)\right\rangle= & -2\left(1+C_{1} \delta+\omega_{1}^{\mu, \delta}\right)\left(b^{\mu, \delta}\right)^{2} \mathrm{e}^{-2\left(1+C_{1} \delta+\omega_{2}^{\mu, \delta}\right)^{1 / 2} T_{j}} \\
& -2 \delta_{j}\left(1+C_{2} \delta+\omega_{3}^{\mu, \delta}\right)\left(d^{\mu, \delta}\right)^{2} \mathrm{e}^{-\left(1+C_{2} \delta+\omega_{4}^{\mu, \delta}\right)^{1 / 2} T_{j}}+O\left(\mathrm{e}^{-3 \lambda_{\mathrm{s}} t}\right)
\end{aligned}
$$

for even values of $k$, where $\omega_{j}^{\mu, \delta}$ denotes a specific function of $\mu$ and $\delta$ which is $O\left(\mu^{2}\right)$. It is convenient to introduce the new variables

$$
r=\mathrm{e}^{-2\left(1+C_{1} \delta+\omega_{2}^{\mu, \delta}\right)^{1 / 2} T}, \quad a_{j}=\mathrm{e}^{-2\left(1+C_{2} \delta+\omega_{4}^{\mu, \delta}\right)^{1 / 2}\left(T_{j}-T\right)},
$$

in terms of which

$\eta_{j}(\boldsymbol{T}, \mu, \delta)=2 \delta_{j}\left(1+C_{1} \delta+\omega_{1}^{\mu, \delta}\right)\left(b^{\mu, \delta}\right)^{2}\left(a_{k} r\right)^{1+\gamma}+2\left(1+C_{2} \delta+\omega_{3}^{\mu, \delta}\right)\left(d^{\mu, \delta}\right)^{2} a_{k} r+\mathcal{S}_{1}(\boldsymbol{a}, \mu, \delta)$

for odd values of $k$ and

$\eta_{j}(\boldsymbol{T}, \mu, \delta)=2\left(1+C_{1} \delta+\omega_{1}^{\mu, \delta}\right)\left(b^{\mu, \delta}\right)^{2}\left(a_{k} r\right)^{1+\gamma}+2 \delta_{j}\left(1+C_{2} \delta+\omega_{3}^{\mu, \delta}\right)\left(d^{\mu, \delta}\right)^{2} a_{k} r+\mathcal{S}_{1}(\boldsymbol{a}, \mu, \delta)$

for even values of $k$, where

$$
\boldsymbol{a}=\left\{a_{j}\right\}_{j=1}^{N-1}, \quad \gamma=\left(\frac{1+C_{1} \delta+\omega_{1}^{\mu, \delta}}{1+C_{2} \delta+\omega_{3}^{\mu, \delta}}\right)^{1 / 2}-1
$$

and $\mathcal{S}_{1}(\boldsymbol{a}, \mu, \delta), \partial_{1} \mathcal{S}_{1}(\boldsymbol{a}, \mu, \delta)=O\left(r^{1+\theta}\right)$ for some fixed positive number $\theta$. A necessary condition for the bifurcation equations

$$
\eta_{j}(\boldsymbol{T}, \mu, \delta)=0, \quad j=1, \ldots, N-1
$$

to have a solution is therefore clearly that $\delta_{j}=-1$.

A straightforward application of the implicit function theorem yields the inverse relation

$$
\delta=\frac{1-(1+\gamma)^{2}}{C_{2}(1+\gamma)^{2}-C_{1}}+O(\mu)
$$


which is well-defined since $C_{2}<C_{1}<0$ (see equation (34)). Defining

$$
c^{\mu, \gamma}=\frac{\left(b^{\mu, \gamma}\right)^{2}\left(1+C_{1} \delta+\omega_{1}^{\mu, \gamma}\right)}{\left(d^{\mu, \gamma}\right)^{2}\left(1+C_{2} \delta+\omega_{3}^{\mu, \gamma}\right)}
$$

and setting $\delta_{j}=-1$, we finally arrive at the equations

$$
a_{j} r\left[-c^{\mu, \gamma}\left(a_{j} r\right)^{\gamma}+1\right]+\mathcal{S}_{1}(\boldsymbol{a}, \mu, \delta(\mu, \gamma))=0,
$$

which are conveniently written in terms of the variables $\rho_{j}:=\left(a_{j} r\right)^{\gamma}$ as

$$
-c^{\mu, \gamma} \rho_{j}+1+\hat{\mathcal{S}}_{1}(\boldsymbol{\rho}, \mu, \gamma)=0,
$$

where $\boldsymbol{\rho}=\left\{\rho_{j}\right\}_{j=1}^{N-1}$ and $\hat{\mathcal{S}}_{1}(\boldsymbol{\rho}, \mu, \gamma), \partial_{1} \hat{\mathcal{S}}_{1}(\boldsymbol{\rho}, \mu, \gamma)=O\left(\rho^{\theta / \gamma}\right)$.

Proposition 4.4 For each sufficiently small value of $\mu$ equations (63) have a local branch of solutions $\rho=\rho(\gamma)$ for $\gamma>0$ which satisfies $\rho_{j}(0)=1 / c_{\mu, 0}$.

Proof Define $\mathcal{F}(\rho, \delta)=\left\{-c^{\mu, \gamma} \rho_{j}+1\right\}_{j=1}^{N-1}+\hat{\mathcal{S}}(\boldsymbol{\rho}, \mu, \delta)$, and note that

$$
\mathcal{F}\left(\left\{1 / c^{\mu, 0}\right\}_{j=1}^{N-1}, 0\right)=\{0\}_{j=1}^{N-1}, \quad \mathrm{~d}_{1} \mathcal{F}\left[\left\{1 / c^{\mu, 0}\right\}_{j=1}^{N-1}, 0\right]=\left\{-c^{\mu, 0}\right\}_{j=1}^{N-1},
$$

where we have used the fact that $\gamma>0$ to show that $\hat{\mathcal{S}}(0, \mu, 0)$ and $\partial_{1} \hat{\mathcal{S}}(0, \mu, 0)$ both vanish; note also that $c^{\mu, 0}>1$ because $c^{0,0}>1$. The result follows from an application of the implicit function theorem.

Our final result follows from the above proposition and the fact that $\operatorname{sgn} \delta=\operatorname{sgn} \gamma$ (see equation (62), bearing in mind that $C_{2}<C_{1}<0$ ).

Theorem 4.5 Choose $N \geq 2$. For sufficiently small values of $\mu \geq 0$ there exists, for sufficiently small values of $\delta>0$, a multipulse homoclinic solution to equations (39)-(42) whose $N$ excursions away from the origin lie close to those of $u_{k, k+1}^{\mu, \delta}$ and $T_{1} u_{k, k+1}^{\mu, \delta}$ (odd values of $k$ ) or $u_{k, k+1}^{\mu, \delta}$ and $T_{2} u_{k, k+1}^{\mu, \delta}$ (even values of $k$ ) in a strictly alternating sequence.

Finally note that the other homoclinic orbits detected near $P_{k, k+1}$ (one for $k=1$ and two for $k \geq 2$ ) cannot be included in the catalogue of basic primary homoclinic orbits. Computing the expansion analogous to (61) for these orbits, one finds that one of $b^{0, \delta}$ and $d^{0, \delta}$ vanishes, so that $\eta_{j}(\mathbf{T}, \mu, \delta)$ is always single-signed and no multipulse homoclinic bifurcation is detected.

\section{Appendix A Formulae for the coefficients in the reduced Hamiltonians}

The coefficients in the Hamiltonian functions discussed in Sections 3.2 and 3.3 are obtained from the formulae

$$
\begin{aligned}
c & =-\frac{\pi \nu^{2}}{\gamma_{1}^{2} \gamma_{2}}(4 \sinh 2 \nu+\sinh 4 \nu), \\
C_{1} & =-\frac{2 k(1+2 k) \nu \sinh ^{2} k \nu}{2 k(1+2 k) \nu+4 k(1+k) \operatorname{coth}(1+k) \nu \sinh ^{2} k \nu+(1-2(k-1) k) \sinh 2 k \nu},
\end{aligned}
$$




$$
\begin{aligned}
& \gamma_{k}^{2} \gamma_{k+1}^{2} c_{3}=\frac{4 k^{2}(1+k)^{2} \pi \nu^{4}}{\alpha} \\
& +\pi \nu^{3}((1+k)(k \sinh 2 \nu+\sinh 2 k \nu)+k \sinh 2(1+k) \nu) \\
& {[2 k(1+k) \nu \cosh \nu-2 \nu \sinh k \nu \sinh (1+k) \nu} \\
& \left.+\left(\alpha+\beta \nu^{2}\right)(-\sinh \nu+(1+2 k) \sinh (1+2 k) \nu)\right] \\
& {\left[-\nu \cosh \nu+\left(\alpha+\beta \nu^{2}\right) \sinh \nu\right]^{-1}} \\
& -\pi \nu^{3}((1+2 k)((1+k) \sinh 2 k \nu+k \sinh 2(1+k) \nu)+k(1+k) \sinh 2(1+2 k) \nu) \\
& {\left[-2 k(1+k) \nu \cosh (1+2 k) \nu+2(1+2 k)^{2} \nu \sinh k \nu \sinh (1+k) \nu\right.} \\
& \left.+\left(\alpha+\beta \nu^{2}(1+2 k)^{2}\right)(\sinh \nu-(1+2 k) \sinh (1+2 k) \nu)\right] \\
& {\left[-(1+2 k) \nu \cosh (1+2 k) \nu+\left(\alpha+\beta \nu^{2}(1+2 k)^{2}\right) \sinh (1+2 k) \nu\right]^{-1}} \\
& +\frac{\pi \nu}{3 k}\left[4 k^{3} \nu\left(3+(1+k)^{2} \nu^{2}\right)+6 k(1+k)^{2} \nu \cosh 2 k \nu\right. \\
& \left.-3\left(1+k\left(2+3 k+2 k(1+k)^{2} \nu^{2}\right)\right) \sinh 2 k \nu\right] \sinh ^{2}(1+k) \nu \\
& +\frac{\pi \nu}{3(k+1)}\left[2(1+k)^{3} \nu\left(6+k^{2}(2-9 \beta) \nu^{2}\right)\right. \\
& +6 k^{2}(1+k) \nu\left(1+3(1+k)^{2} \beta \nu^{2}\right) \cosh 2(1+k) \nu \\
& \left.-3\left(2+k\left(4+3 k+2 k(1+k)^{2} \nu^{2}\right)\right) \sinh 2(1+k) \nu\right] \sinh ^{2} k \nu \\
& +\frac{48 \pi \nu}{3(1+2 k)^{3}} k^{2}(1+k)^{2} \sinh k \nu \sinh (1+k) \nu \\
& {\left[-2(1+2 k)^{3} \nu \cosh \nu+2(1+2 k) \nu \cosh (1+2 k) \nu\right.} \\
& \left.+(1+2 k)^{3}\left(2+\nu^{2}\right) \sinh \nu-\left(2+\nu^{2}(1+2 k)^{2}\right) \sinh (1+2 k) \nu\right] \\
& +\frac{\pi \nu}{12 k(1+k)(1+2 k)^{3}} \\
& {[-24 k(1+k)(1+2 k)(1+2 k(1+k))(-1+4 k(-1+k(1+2 k(2+k)))) \nu} \\
& +16 k^{3}(1+k)^{3}(1+2 k)^{3} \nu^{3}-3 \sinh 2 \nu-6 \sinh 2 k \nu+3 \sinh 2(1+2 k) \nu \\
& -6 k(1+k)(1+2 k)^{3}(1+2 k(1+k)(1+8 k(1+k))) \nu \cosh 2 \nu \\
& +k\left[-4(1+k)^{3}(1+2 k) \nu\left(3+2 k\left(6+k\left(-6(3+8 k(1+k))+(\nu+2 k \nu)^{2}\right)\right)\right) \cosh 2 k \nu\right. \\
& -4 k^{2}(1+k)(1+2 k) \nu(-3(15+4 k(15+k(27+8 k(3+k)))) \\
& \left.+2(1+k)^{2}(1+2 k)^{2} \nu^{2}\right) \cosh 2(1+k) \nu \\
& -6(1+k)(1+2 k)\left(1+6 k(1+k)(1+2 k)^{2}\right) \nu \cosh 2(1+2 k) \nu \\
& +3\left(-7+k\left(-19+6 k+268 k^{2}+1048 k^{3}+2016 k^{4}+2112 k^{5}+1152 k^{6}+256 k^{7}\right.\right. \\
& \left.\left.+2(1+k)^{2}(1+2 k)^{3}(3+8 k(1+k)) \nu^{2}\right)\right) \sinh 2 \nu \\
& +6\left(-9+k\left(-35-77 k-6 k^{2}+404 k^{3}+968 k^{4}+1056 k^{5}+576 k^{6}+128 k^{7}\right.\right. \\
& \left.\left.+2(1+k)^{3}(-1+2 k)(1+4 k)(\nu+2 k \nu)^{2}\right)\right) \sinh 2 k \nu \\
& -6\left(2+k\left(16+k\left(83+274 k+644 k^{2}+1048 k^{3}+1056 k^{4}+576 k^{5}+128 k^{6}\right.\right.\right. \\
& \left.\left.\left.+2(1+k)^{2}(1+2 k)^{2}(3+2 k)(3+4 k) \nu^{2}\right)\right)\right) \sinh 2(1+k) \nu \\
& -3(1+k)\left(-11+2 k(1+k)\left(-20+3 \nu^{2}\right.\right. \\
& \left.\left.\left.\left.+8 k(1+k)\left(-5+2\left(1+k+k^{2}\right) \nu^{2}\right)\right)\right) \sinh 2(1+2 k) \nu\right]\right],
\end{aligned}
$$




$$
\begin{aligned}
\gamma_{k}^{4} c_{1}=k \pi \nu[ & \alpha\left(4 \alpha-2 k^{2}(2+3 \alpha-10 \beta) \nu^{2}-8 k^{4}(-1+3 \beta) \nu^{4}\right) \\
& -\left(5 \alpha^{2}+2 k^{2} \alpha(-2+\alpha+10 \beta) \nu^{2}+8 k^{4}(4+\alpha(4+\beta)) \nu^{4}\right) \cosh 2 k \nu \\
& +2 \alpha\left(-5 \alpha+2 k^{2}(-2+3 \alpha-10 \beta) \nu^{2}+8 k^{4}(1+3 \beta) \nu^{4}\right) \cosh 4 k \nu \\
& +\alpha\left(5 \alpha+2 k^{2}(6+\alpha+10 \beta) \nu^{2}+8 k^{4} \beta \nu^{4}\right) \cosh 6 k \nu \\
& +2 k \nu\left(5(-1+\alpha) \alpha+4 k^{2} \alpha(10+2 \alpha+5 \beta) \nu^{2}+32 k^{4}(1+\alpha) \beta \nu^{4}\right) \sinh 2 k \nu \\
& +4 k \alpha \nu\left(5+\alpha-2 k^{2}(-1+\alpha-2 \beta) \nu^{2}-8 k^{4} \beta \nu^{4}\right) \sinh 4 k \nu \\
& \left.-2 k \alpha \nu\left(5+3 \alpha+12 k^{2} \beta \nu^{2}\right) \sinh 6 k \nu\right] \\
& {\left[-8 k \alpha \nu \cosh 2 k \nu+4 \alpha\left(\alpha+4 k^{2} \beta \nu^{2}\right) \sinh 2 k \nu\right]^{-1}, }
\end{aligned}
$$

where $(\beta, \alpha)=P_{k, k+1}$ and $\gamma_{k}, \gamma_{k+1}$ are given by (28). The coefficient $C_{2}$ is given by the formula for $C_{1}$ with $k$ replaced by $k+1$ and the coefficients $c_{2}, d$ are given by the formula for $c_{1}$ with $k$ replaced by respectively $k+1$ and 2 .

\section{References}

[1] Buffoni, B. \& Groves, M. D. 1999 A multiplicity result for solitary gravity-capillary waves in deep water via critical-point theory. Arch. Rat. Mech. Anal. 146, 183-220.

[2] Buffoni, B., Groves, M. D. \& Toland, J. F. 1996 A plethora of solitary gravitycapillary water waves with nearly critical Bond and Froude numbers. Phil. Trans. Roy. Soc. Lond. A 354, 575-607.

[3] Cotter, C. 1986 The $1: 1$ semisimple resonance. (Doctoral thesis, University of California at Santa Cruz)

[4] Craig, W. \& Nicholls, D. P. 2000 Traveling two and three dimensional capillary gravity water waves. SIAM J. Math. Anal. 32, 323-359.

[5] Devaney, R. L. 1976 Homoclinic orbits in Hamiltonian systems. J. Diff. Eqns. 21, 431438.

[6] Groves, M. D. 2001 An existence theory for three-dimensional periodic travelling gravity-capillary water waves with bounded transverse profiles. Physica D 152-153, 395415.

[7] Groves, M. D. \& Haragus, M. 2003 A bifurcation theory for three-dimensional oblique travelling gravity-capillary water waves. J. Nonlinear Sci. 13, 397-447.

[8] Groves, M. D., Haragus, M. \& Sun, S.-M. 2002 A dimension-breaking phenomenon in the theory of gravity-capillary water waves. Phil. Trans. Roy. Soc. Lond. A 360, 21892243.

[9] Groves, M. D. \& Mielke, A. 2001 A spatial dynamics approach to three-dimensional gravity-capillary steady water waves. Proc. Roy. Soc. Edin. A 131, 83-136. 
[10] Haragus-Courcelle, M. \& Ilichev, A. 1998 Three-dimensional solitary waves in the presence of additional surface effects. Eur. J. Mech. B. Fluids 17, 739-768.

[11] Haragus-Courcelle, M. \& Pego, R. L. 2000 Spatial wave dynamics of steady oblique wave interactions. Physica D 145, 207-232.

[12] IoOss, G. \& KIRChGÄSsner, K. 1990 Bifurcation d'ondes solitaires en présence d'une faible tension superficielle. C. R. Acad. Sci. Paris, Sér. 1 311, 265-268.

[13] Iooss, G. \& KirchGässner, K. 1992 Water waves for small surface tension: an approach via normal form. Proc. Roy. Soc. Edin. A 122, 267-299.

[14] Jones, M. C. W. 1989 Small amplitude gravity-capillary waves in a channel of finite depth. Glasgow Math. J. 31, 141-160.

[15] Kato, T. 1976 Perturbation Theory for Linear Operators, 2nd edn. New York: SpringerVerlag.

[16] KIRCHGÄSSNER, K. 1982 Wave solutions of reversible systems and applications. J. Diff. Eqns. 45, 113-127.

[17] KIRCHG ÄSSNER, K. 1988 Nonlinear resonant surface waves and homoclinic bifurcation. Adv. Appl. Math. 26, 135-181.

[18] Lin, X. B. 1990 Using Melnikov's method to solve Shilnikov's problems. Proc. Roy. Soc. Edin. A 116, 295-325.

[19] Mielke, A. 1988 Reduction of quasilinear elliptic equations in cylindrical domains with applications. Math. Meth. Appl. Sci. 10, 51-66.

[20] Mielke, A. 1991 Hamiltonian and Lagrangian Flows on Center Manifolds. Berlin: Springer-Verlag.

[21] S Andstede, B. 1993 Verzweigungstheorie homokliner Verdopplungen. (Doctoral thesis, Universität Stuttgart)

[22] Sandstede, B., Jones, C. K. R. T. \& Alexander, J. C. 1997 Existence and stability of $N$-pulses on optical fibers with phase-sensitive amplifiers. Physica D 106, 167-206.

[23] Stoker, J. J. 1957 Water Waves: The Mathematical Theory with Applications. New York: Interscience.

[24] YEW, A. C. 2001 Multipulses of nonlinearly coupled Schrödinger equations. J. Diff. Eqns. 173, 92-137.

[25] Yew, A. C., Sandstede, B. \& Jones, C. K. R. T. 2000 Instability of multiple pulses in nonlinear Schrödinger equations. Phys. Rev. E 61, 5886-5892. 